\title{
Global subelliptic estimates for Kramers-Fokker-Planck operators with some class of polynomials
}

\author{
Mona Ben Said \\ Laboratoire Analyse, Géométrie et Applications \\ Université Paris 13 \\ 99 Avenue Jean Baptiste Clément \\ 93430 Villetaneuse, France \\ bensaid@univ-paris13.fr
}

May 27, 2019

\begin{abstract}
In this article we study some Kramers-Fokker-Planck operators with a polynomial potential $V(q)$ of degree greater than two having quadratic limiting behavior. This work provides an accurate global subelliptic estimate for KFP operators under some conditions imposed on the potential.
\end{abstract}

Key words: subelliptic estimates, compact resolvent, Kramers-Fokker-Planck operator. MSC-2010: 35Q84, 35H20, 35P05, 47A10, 14P10

\section{Contents}

1 Introduction and main results 2

2 Preliminary results $\quad 5$

3 Proof of Theorem $1.1 \quad 14$

4 Applications $\quad 24$

$\begin{array}{ll}\text { Appendix A Slow metric, partition of unity } & 29\end{array}$

$\begin{array}{lll}\text { Appendix B Around Tarski-Seidenberg theorem } & 34\end{array}$ 


\section{Introduction and main results}

The Kramers-Fokker-Planck operator reads

$$
K_{V}=p . \partial_{q}-\partial_{q} V(q) . \partial_{p}+\frac{1}{2}\left(-\Delta_{p}+p^{2}\right), \quad(q, p) \in \mathbb{R}^{2 d},
$$

where $q$ denotes the space variable, $p$ denotes the velocity variable, $x . y=\sum_{j=1}^{d} x_{j} y_{j}, x^{2}=\sum_{j=1}^{d} x_{j}^{2}$ and the potential $V(q)=\sum_{|\alpha| \leq r} V_{\alpha} q^{\alpha}$ is a real-valued polynomial function on $\mathbb{R}^{d}$ with $d^{\circ} V=r$.

There have been several works concerned with the operator $K_{V}$ with diversified approaches. In this article we impose some kind of assumptions on the polynomial potential $V(q)$, so that the Kramers-Fokker-Planck operator $K_{V}$ admits a global subelliptic estimate and has a compact resolvent. This problem is closely related to the return to the equilibrium for the Kramers-Fokker-Planck operator (see [HeNi][Nie][Nou]). As mentioned in [HerNi] and [Nie], the analysis of $K_{V}$ is also strongly linked to the one of the Witten Laplacian $\Delta_{V}^{(0)}=-\Delta_{q}+|\nabla V(q)|^{2}-\Delta V(q)$. This relation yielded to the following conjecture established by Helffer-Nier:

$$
\left(1+K_{V}\right)^{-1} \text { compact } \Leftrightarrow\left(1+\Delta_{V}^{(0)}\right)^{-1} \text { compact . }
$$

This conjecture has been partially resolved in simple cases (see for example [HeNi], [HerNi] and $[\mathrm{Li}]$ ), whereas for the operator $\Delta_{V}^{(0)}$ very general criteria of compactness work for polynomial potiential $V(q)$ of arbitrary degree. These last criteria require an analysis of the degeneracies at infinity of the potential and rely on extremely sophisticated tools of hypoellipticity developed by Helffer and Nourrigat in the 1980's (see [HeNo], [Nie]). Among the particularities of these last analysis, we mention that the compactness results obtained for degenerate potentials at infiniy were not the same for $\Delta_{+V}^{(0)}$ as $\Delta_{-V}^{(0)}$. The typical example which was considered is the case $V\left(q_{1}, q_{2}\right)=q_{1}^{2} q_{2}^{2}$ in dimension $d=2$ : The operator $\Delta_{-V}^{(0)}$ has a compact resolvent, while $\Delta_{+V}^{(0)}$ has not.

In the case of the Kramers-Fokker-Planck operator, there have been extensive works concerned with the case $d^{\circ} V \leq 2$ (see [Hor][HiPr][Vio][Vio1][AlVi][BNV]). Nevertheless, as far as general potential is concerned, different kind of sufficient conditions on $V(q)$ had been examined by Hérau-Nier [HerNi], Helffer-Nier [HeNi], Villani [Vil] and Wei-Xi Li [Li]. These first results considered only variants of the elliptic situation at the infinity ( for non-degenerate potential), which did not distinguish the sign $\pm V(q)$. Lately a significant improvement of those works has been done by Wei-Xi Li [Li2] based on some multipliers methods. In [Li2], Wei-Xi Li showed that for potentials similar to $V\left(q_{1}, q_{2}\right)=q_{1}^{2} q_{2}^{2}$ the results for $K_{ \pm V}$ were the same as for $\Delta_{ \pm V}^{(0)}$, thus comforting the idea that the conjecture (1.2) is true.

The ultimate goal would be to develop a complete recurrence with respect to $d^{\circ} V$ for the Kramers-Fokker-Planck operator like it is possible to do for the Witten Laplacian as recalled in [HeNi] (cf. Teorem 10.16 page 106) and [Nie] by following the general approach of HelfferNourrigat in $[\mathrm{HeNo}]$ and $[\mathrm{Nou}]$. Although we are not able to write a complete induction, we 
establish here subelliptic estimates for $K_{V}$ for a rather general class of polynomial potentials with criteria which distinguish clearly the sign $\pm V(q)$. The asymtotic behaviour of those polynomials is governed by at most quadratic parameter dependent potentials, and the global subelliptic estimates in which arise some logarithmic weights are know to be essentially optimal in the quadratic case (see $[\mathrm{BNV}])$.

Denoting

$$
O_{p}=\frac{1}{2}\left(D_{p}^{2}+p^{2}\right)
$$

and

$$
X_{V}=p . \partial_{q}-\partial_{q} V(q) . \partial_{p},
$$

we can rewrite the Kramers-Fokker-Planck operator $K_{V}$ defined in (1.1) as $K_{V}=X_{V}+O_{p}$. Notations: Throughout the paper we use the notation

$$
\langle\cdot\rangle=\sqrt{1+|\cdot|^{2}} .
$$

For an arbitrary polynomial $V(q)$ of degree $r$, we denote for all $q \in \mathbb{R}^{d}$

$$
\begin{aligned}
& \operatorname{Tr}_{+, V}(q)=\sum_{\substack{\nu \in \operatorname{Spec}(\operatorname{Hess} V) \\
\nu>0}} \nu(q), \\
& \operatorname{Tr}_{-, V}(q)=-\sum_{\substack{\nu \in \operatorname{Spec}(\operatorname{Hess} V) \\
\nu \leq 0}} \nu(q) .
\end{aligned}
$$

Futhermore, for a polynomial $P \in E_{r}:=\left\{P \in \mathbb{R}\left[X_{1}, \ldots, X_{d}\right], d^{\circ} P \leq r\right\}$ and all natural number $n \in\{1, \ldots, r\}$, we define the functions $R_{P}^{\geq n}: \mathbb{R}^{d} \rightarrow \mathbb{R}$ and $R_{P}^{=n}: \mathbb{R}^{d} \rightarrow \mathbb{R}$ by

$$
\begin{aligned}
& R_{P}^{\geq n}(q)=\sum_{n \leq|\alpha| \leq r}\left|\partial_{q}^{\alpha} P(q)\right|^{\frac{1}{|\alpha|}} \\
& R_{P}^{=n}(q)=\sum_{|\alpha|=n}\left|\partial_{q}^{\alpha} P(q)\right|^{\frac{1}{|\alpha|}}
\end{aligned}
$$

For arbitrary real functions $A$ and $B$, we make also use of the following notation

$$
A \asymp B \Longleftrightarrow \exists c \geq 1: c^{-1}|B| \leq|A| \leq c|B| .
$$

This work is essentially based on the recent publication by Ben Said, Nier, and Viola [BNV], which deals with the analysis of Kramers-Fokker-Planck operators with polynomials of degree less than 3. In this case we define the constants $A_{V}$ and $B_{V}$ by

$$
\begin{aligned}
& A_{V}=\max \left\{\left(1+\operatorname{Tr}_{+, V}\right)^{2 / 3}, 1+\operatorname{Tr}_{-, V}\right\}, \\
& B_{V}=\max \left\{\min _{q \in \mathbb{R}^{d}}|\nabla V(q)|^{4 / 3}, \frac{1+\operatorname{Tr}_{-, V}}{\log \left(2+\operatorname{Tr}_{-, V}\right)^{2}}\right\} .
\end{aligned}
$$


As proved in $[\mathrm{BNV}]$, there is a constant $c>0$ such that the following global subelliptic estimate with remainder

$$
\begin{aligned}
\left\|K_{V} u\right\|_{L^{2}\left(\mathbb{R}^{2 d}\right)}^{2}+A_{V}\|u\|_{L^{2}\left(\mathbb{R}^{2 d}\right)}^{2} \geq c\left(\left\|O_{p} u\right\|_{L^{2}\left(\mathbb{R}^{2 d}\right)}^{2}\right. & +\left\|X_{V} u\right\|_{L^{2}\left(\mathbb{R}^{2 d}\right)}^{2} \\
& \left.+\left\|\left\langle\partial_{q} V(q)\right\rangle^{2 / 3} u\right\|_{L^{2}\left(\mathbb{R}^{2 d}\right)}^{2}+\left\|\left\langle D_{q}\right\rangle^{2 / 3} u\right\|_{L^{2}\left(\mathbb{R}^{2 d}\right)}\right)
\end{aligned}
$$

holds for all $u \in \mathcal{C}_{0}^{\infty}\left(\mathbb{R}^{2 d}\right)$. Moreover, if $V$ does not have any local minimum, that is if $\operatorname{Tr}_{-, V}+\min _{q \in \mathbb{R}^{d}}|\nabla V(q)| \neq 0$, there exists a constant $c>0$ such that

$$
\left\|K_{V} u\right\|_{L^{2}\left(\mathbb{R}^{2 d}\right)}^{2} \geq c B_{V}\|u\|_{L^{2}\left(\mathbb{R}^{2 d}\right)}^{2},
$$

holds for all $u \in \mathcal{C}_{0}^{\infty}\left(\mathbb{R}^{2 d}\right)$. Hence combining (1.6) and (1.5), there is a constant $c>0$ so that

$$
\begin{aligned}
\left\|K_{V} u\right\|_{L^{2}\left(\mathbb{R}^{2 d}\right)}^{2} \geq \frac{c}{1+\frac{A_{V}}{B_{V}}}\left(\left\|O_{p} u\right\|_{L^{2}\left(\mathbb{R}^{2 d}\right)}^{2}\right. & +\left\|X_{V} u\right\|_{L^{2}\left(\mathbb{R}^{2 d}\right)}^{2} \\
& \left.+\left\|\left\langle\partial_{q} V(q)\right\rangle^{2 / 3} u\right\|_{L^{2}\left(\mathbb{R}^{2 d}\right)}^{2}+\left\|\left\langle D_{q}\right\rangle^{2 / 3} u\right\|_{L^{2}\left(\mathbb{R}^{2 d}\right)}\right)
\end{aligned}
$$

is valid for all $u \in \mathcal{C}_{0}^{\infty}\left(\mathbb{R}^{2 d}\right)$. The constants appearing in (1.5), (1.6) and (1.7) are independent of the potential $V$. We recall here that for a smooth potential $V \in \mathcal{C}^{\infty}\left(\mathbb{R}^{d}\right)$, our operator $K_{V}$ is essential maximal accretive when endowed with the domain $\mathcal{C}_{0}^{\infty}\left(\mathbb{R}^{2 d}\right)$ [HeNi] (cf. Proposition 5.5 page 44$)$. As a result the domain of its closure is given by

$$
D\left(K_{V}\right)=\left\{u \in L^{2}\left(\mathbb{R}^{2 d}\right), K_{V} u \in L^{2}\left(\mathbb{R}^{2 d}\right)\right\} .
$$

Consequently by density of $\mathcal{C}_{0}^{\infty}\left(\mathbb{R}^{2 d}\right)$ in $D\left(K_{V}\right)$ all estimates stated in this paper, which are checked with $C_{0}^{\infty}\left(\mathbb{R}^{2 d}\right)$ functions, can be extended to the domain of $K_{V}$.

Given a polynomial $V(q)$ with degree $r$ greater than two, our result will require the following assumption after setting for $\kappa>0$

$$
\Sigma(\kappa)=\left\{q \in \mathbb{R}^{d},|\nabla V(q)|^{\frac{4}{3}} \geq \kappa\left(|\operatorname{Hess} V(q)|+R_{V}^{\geq 3}(q)^{4}+1\right)\right\} .
$$

Assumption 1. There exist large constants $\kappa_{0}, C_{1}>1$ such that for all $\kappa \geq \kappa_{0}$ the polynomial $V(q)$ satisfies the following properties

$$
\operatorname{Tr}_{-, V}(q) \geq \frac{1}{C_{1}} \operatorname{Tr}_{+, V}(q), \text { for all } q \in \mathbb{R}^{d} \backslash \Sigma(\kappa) \text { with }|q| \geq C_{1}
$$

moreover if $\mathbb{R}^{d} \backslash \Sigma(\kappa)$ is not bounded

$$
\lim _{\substack{q \rightarrow \infty \\ q \in \mathbb{R}^{d} \backslash \Sigma(\kappa)}} \frac{R_{V}^{\geq 3}(q)^{4}}{|\operatorname{Hess} V(q)|}=0 .
$$


Our main result is the following.

Theorem 1.1. Let $V(q)$ be a polynomial of degree $r$ greater than two verifying Assumption 1. Then there exists a strictly positive constant $C_{V}>1$ (depending on $V$ ) such that

$$
\begin{aligned}
\left\|K_{V} u\right\|_{L^{2}}^{2}+C_{V}\|u\|_{L^{2}}^{2} \geq \frac{1}{C_{V}}\left(\left\|L\left(O_{p}\right) u\right\|_{L^{2}}^{2}\right. & +\left\|L\left(\langle\nabla V(q)\rangle^{\frac{2}{3}}\right) u\right\|_{L^{2}}^{2} \\
& \left.+\left\|L\left(\langle\operatorname{Hess} V(q)\rangle^{\frac{1}{2}}\right) u\right\|_{L^{2}}^{2}+\left\|L\left(\left\langle D_{q}\right\rangle^{\frac{2}{3}}\right) u\right\|_{L^{2}}^{2}\right),
\end{aligned}
$$

holds for all $u \in D\left(K_{V}\right)$ where $L(s)=\frac{s+1}{\log (s+1)}$ for any $s \geq 1$.

Corollary 1.2. If $V(q)$ is polynomial of degree greater than two that satisfies Assumption 1, then the Kramers-Fokker-Planck operator $K_{V}$ has a compact resolvent.

Proof. Proof of Corollary 1.2

Assume $0<\delta<1$. Define the functions $f_{\delta}: \mathbb{R}^{d} \rightarrow \mathbb{R}$ by

$$
f_{\delta}(q)=|\nabla V(q)|^{\frac{4}{3}(1-\delta)}+|\operatorname{Hess} V(q)|^{1-\delta} .
$$

From (1.10) in Theorem 1.1 there is a constant $C_{V}>1$ such that

$$
\left\|K_{V} u\right\|_{L^{2}}^{2}+C_{V}\|u\|_{L^{2}}^{2} \geq \frac{1}{C_{V}}\left(\left\langle u, f_{\delta} u\right\rangle+\left\|L\left(O_{p}\right) u\right\|_{L^{2}}^{2}+\left\|L\left(\left\langle D_{q}\right\rangle^{\frac{2}{3}}\right) u\right\|_{L^{2}}^{2}\right)
$$

holds for all $u \in \mathcal{C}_{0}^{\infty}\left(\mathbb{R}^{2 d}\right)$ and all $\delta \in(0,1)$. In order to prove that the operator $K_{V}$ has a compact resolvent it is sufficient to show that $\lim _{q \rightarrow+\infty} f_{\delta}(q)=+\infty$.

To do so, assume $A>0$ and denote $\kappa=A^{\frac{1}{1-\delta}}$. If $q \in \Sigma(\kappa)$, one has

$$
|\nabla V(q)|^{\frac{4}{3}(1-\delta)} \geq \kappa^{1-\delta}=A .
$$

Else if $q \in \mathbb{R}^{d} \backslash \Sigma(\kappa)$ by (1.9) in Assumption 1, $\lim _{\substack{q \rightarrow \infty \\ q \in \mathbb{R}^{d} \backslash \Sigma(\kappa)}}|\operatorname{Hess} V(q)|=+\infty$. Hence there exists a constant $\eta>0$ such that $|\operatorname{Hess} V(q)|^{1-\delta} \geq A$ for all $q \in \mathbb{R}^{d} \backslash \Sigma(\kappa)$ with $|q| \geq \eta$.

Remark 1.3. The results of Theorem 1.1 and Corollary 1.2 can be extended in the case when $V=V_{1}+V_{2}$ where $V_{1}$ is polynomial satisfying Assumption 1 and $V_{2}$ is a function in $\mathcal{S}\left(\mathbb{R}^{d}\right)$.

\section{Preliminary results}

This work is essentially based on two main strategies. The first one consists in the use of a partition of unity which is the most important tool that allows one to pass from local to global estimates. 
In this paper, given a polynomial $V(q)$ we make use of a locally finite partition of unity with respect to the position variable $q \in \mathbb{R}^{d}$

$$
\sum_{j \in \mathbb{N}} \chi_{j}^{2}(q)=\sum_{j \in \mathbb{N}} \widetilde{\chi}_{j}^{2}\left(R_{V}^{23}\left(q_{j}\right)^{-1}\left(q-q_{j}\right)\right)=1
$$

where

$$
\operatorname{supp} \tilde{\chi}_{j} \subset B\left(q_{j}, a\right) \text { and } \tilde{\chi}_{j} \equiv 1 \text { in } B\left(q_{j}, b\right)
$$

for some $q_{j} \in \mathbb{R}^{d}$ with $0<b<a$ independent of $j \in \mathbb{N}$. Such a partition is described more precisely in Lemma A.7 after taking $n=3$. In our study introducing this partition yields to errors to be well controlled.

The second approach lies in the decomposition of the operator $K_{V}$ onto two parts so that the first one be a Kramers-Fokker-Planck operator with polynomial potential of degree less than three. On this way, based on [BNV], we derive the result of Theorem 1.1.

In order to prove Theorem 1.1 we need the following basic lemmas.

Lemma 2.1. Assume $V \in E_{r}$ with degree $r \in \mathbb{N}$. Consider the Kramers-Fokker-Planck operator $K_{V}$ defined as in (1.1). For a locally finite partition of unity namely $\sum_{j \in \mathbb{N}} \chi_{j}^{2}(q)=1$ one has

$$
\left\|K_{V} u\right\|_{L^{2}\left(\mathbb{R}^{2 d}\right)}^{2}=\sum_{j \in \mathbb{N}}\left\|K_{V}\left(\chi_{j} u\right)\right\|_{L^{2}\left(\mathbb{R}^{2 d}\right)}^{2}-\left\|\left(p \partial_{q} \chi_{j}\right) u\right\|_{L^{2}\left(\mathbb{R}^{2 d}\right)}^{2},
$$

for all $u \in \mathcal{C}_{0}^{\infty}\left(\mathbb{R}^{2 d}\right)$.

In particular when the degree of $V$ is larger than two and the cutoff functions $\chi_{j}$ have the form (2.1), there exists a constant $c_{d}>0$ (depending on the dimension d) so that

$$
\left\|K_{V} u\right\|_{L^{2}\left(\mathbb{R}^{2 d}\right)}^{2} \geq \sum_{j \in \mathbb{N}}\left\|K_{V}\left(\chi_{j} u\right)\right\|_{L^{2}\left(\mathbb{R}^{2 d}\right)}^{2}-c_{d} R_{V}^{23}\left(q_{j}\right)^{2}\left\|p \chi_{j} u\right\|_{L^{2}\left(\mathbb{R}^{2 d}\right)}^{2}
$$

holds for all $u \in \mathcal{C}_{0}^{\infty}\left(\mathbb{R}^{2 d}\right)$.

Proof. First let $V \in E_{r}$ with $r \in \mathbb{N}$ is the degree of $V$. Assume that $u \in \mathcal{C}_{0}^{\infty}\left(\mathbb{R}^{2 d}\right)$. On the one hand,

$$
\left\|K_{V} u\right\|_{L^{2}}^{2}=\sum_{j \in \mathbb{N}}\left\langle K_{V} u, \chi_{j}^{2} K_{V} u\right\rangle=\sum_{j \in \mathbb{N}}\left\langle u, K_{V}^{*} \chi_{j}^{2} K_{V} u\right\rangle
$$

On the other hand,

$$
\sum_{j \in \mathbb{N}}\left\|K_{V}\left(\chi_{j} u\right)\right\|_{L^{2}}^{2}=\sum_{j \in \mathbb{N}}\left\langle u, \chi_{j} K_{V}^{*} K_{V} \chi_{j} u\right\rangle
$$

Putting the above equalities together

$$
\left\|K_{V} u\right\|_{L^{2}}^{2}-\sum_{j \in \mathbb{N}}\left\|K_{V}\left(\chi_{j} u\right)\right\|_{L^{2}}^{2}=\sum_{j \in \mathbb{N}}\left\langle u,\left(K_{V}^{*} \chi_{j}^{2} K_{V}-\chi_{j} K_{V}^{*} K_{V} \chi_{j}\right) u\right\rangle
$$


Using commutators, we compute

$$
\begin{aligned}
K_{V}^{*} \chi_{j}^{2} K_{V} & =K_{V}^{*} \chi_{j}\left[\chi_{j}, K_{V}\right]+K_{V}^{*} \chi_{j} K_{V} \chi_{j} \\
& =K_{V}^{*} \chi_{j}\left[\chi_{j}, K_{V}\right]+\left[K_{V}^{*}, \chi_{j}\right] K_{V} \chi_{j}+\chi_{j} K_{V}^{*} K_{V} \chi_{j} \\
& =K_{V}^{*} \chi_{j}\left[\chi_{j}, K_{V}\right]+\left[K_{V}^{*}, \chi_{j}\right]\left(\left[K_{V}, \chi_{j}\right]+\chi_{j} K_{V}\right)+\chi_{j} K_{V}^{*} K_{V} \chi_{j}
\end{aligned}
$$

Thus

$$
K_{V}^{*} \chi_{j}^{2} K_{V}-\chi_{j} K_{V}^{*} K_{V} \chi_{j}=K_{V}^{*} \chi_{j}\left[\chi_{j}, K_{V}\right]+\left[K_{V}^{*}, \chi_{j}\right] \chi_{j} K_{V}+\left[K_{V}^{*}, \chi_{j}\right] \circ\left[K_{V}, \chi_{j}\right] .
$$

Now it is easy to check the following commutation relations

$$
\left\{\begin{array}{l}
{\left[\chi_{j}, K_{V}\right]=-\left[K_{V}, \chi_{j}\right]=-\left[p \partial_{q}, \chi_{j}(q)\right]=-p \partial_{q} \chi_{j}} \\
{\left[K_{V}^{*}, \chi_{j}\right]=\left[-p \partial_{q}, \chi_{j}(q)\right]=-p \partial_{q} \chi_{j}} \\
{\left[K_{V}^{*}, \chi_{j}\right] \circ\left[K_{V}, \chi_{j}\right]=-\left(p \partial_{q} \chi_{j}\right)^{2}}
\end{array}\right.
$$

Collecting the terms, we obtain

$$
\begin{aligned}
\sum_{j \in \mathbb{N}}\left(K_{V}^{*} \chi_{j}^{2} K_{V}-\chi_{j} K_{V}^{*} K_{V} \chi_{j}\right) & =\sum_{j \in \mathbb{N}} K_{V}^{*} \chi_{j}\left(-p \partial_{q} \chi_{j}\right)+\left(-p \partial_{q} \chi_{j}\right) \chi_{j} K_{V}-\left(p \partial_{q} \chi_{j}\right)^{2} \\
& =\sum_{j \in \mathbb{N}} K_{V}^{*}\left(\partial_{q}\left(\frac{\chi_{j}^{2}}{2}\right)\right)-p \partial_{q}\left(\frac{\chi_{j}^{2}}{2}\right) K_{V}-\left(p \partial_{q} \chi_{j}\right)^{2} \\
=-\left(p \partial_{q} \chi_{j}\right)^{2} &
\end{aligned}
$$

where in the last line we make use simply $\sum_{j \in \mathbb{N}} \chi_{j}^{2}(q)=1$.

From this follows immediately the identity

$$
\left\|K_{V} u\right\|_{L^{2}}^{2}=\sum_{j \in \mathbb{N}}\left(\left\|K_{V}\left(\chi_{j} u\right)\right\|_{L^{2}}^{2}-\left\|\left(p \partial_{q} \chi_{j}\right) u\right\|_{L^{2}}^{2}\right)
$$

for all $u \in \mathcal{C}_{0}^{\infty}\left(\mathbb{R}^{2 d}\right)$.

Next, suppose that the degree of $V$ is greater than two and $\chi_{j}(q)=\tilde{\chi}_{j}\left(R_{V}^{\geq 3}\left(q_{j}\right)^{-1}\left(q-q_{j}\right)\right)$ for all index $j$ and any $q \in \mathbb{R}^{d}$ with

$$
\operatorname{supp} \tilde{\chi}_{j} \subset B\left(q_{j}, a\right) \text { and } \tilde{\chi}_{j} \equiv 1 \text { in } B\left(q_{j}, b\right) \text {. }
$$

Then we can write

$$
\begin{aligned}
\sum_{j \in \mathbb{N}}\left\|\left(p \partial_{q} \chi_{j}\right) u\right\|^{2} & =\sum_{j \in \mathbb{N}} \sum_{j^{\prime} \in \mathbb{N}}\left\|\left(p \partial_{q} \chi_{j}\right) \chi_{j^{\prime}} u\right\|^{2} \\
& \leq c_{d} \sum_{j \in \mathbb{N}} R_{V}^{\geq 3}\left(q_{j}\right)^{2}\left\|p \chi_{j} u\right\|^{2}
\end{aligned}
$$

where $c_{d}$ is a constant that depends only on the dimension $d$. Here the last inequality is due to the fact that for each index $j$ there are finitely many $j^{\prime}$ such that $\left(\partial_{q} \chi_{j}\right) \chi_{j^{\prime}}$ is nonzero. 
Before stating the following lemma, we fix and remind some notations.

Notations 2.2. Let $V$ be a polynomial of degree $r$ larger than two. Consider a locally finite partition of unity $\sum_{j \in \mathbb{N}} \chi_{j}^{2}(q)=1$ described as in (2.1).

Set for all $\kappa>0$

$$
J(\kappa)=\left\{j \in \mathbb{N}, \text { such that } \operatorname{supp} \chi_{j} \subset \Sigma(\kappa)\right\}
$$

where we recall that

$$
\Sigma(\kappa)=\left\{q \in \mathbb{R}^{d},|\nabla V(q)|^{\frac{4}{3}} \geq \kappa\left(|\operatorname{Hess} V(q)|+R_{V}^{\geq 3}(q)^{4}+1\right)\right\} .
$$

For a given $\kappa>0$ and all index $j \in \mathbb{N}$, let $V_{j}^{2}$ be the polynomial of degree less than three given by

$$
V_{j}^{2}(q)=\sum_{0 \leq|\alpha| \leq 2} \frac{\partial_{q}^{\alpha} V\left(q_{j}^{\prime}\right)}{\alpha !}\left(q-q_{j}^{\prime}\right)^{\alpha}
$$

where

$$
\begin{cases}q_{j}^{\prime}=q_{j} & \text { if } j \in J(\kappa) \\ q_{j}^{\prime} \in\left(\operatorname{supp} \chi_{j}\right) \cap\left(\mathbb{R}^{d} \backslash \Sigma(\kappa)\right) & \text { else. }\end{cases}
$$

Lemma 2.3. Assume $V$ a polynomial of degree $r$ larger than two. Consider a locally finite partion of unity described as in (2.1). For a multi-index $\alpha \in \mathbb{N}^{d}$ of length $|\alpha| \in\{1,2\}$ and all $j \in \mathbb{N}$, one has

$$
\left|\partial_{q}^{\alpha} V(q)-\partial_{q}^{\alpha} V_{j}^{2}(q)\right| \leq c_{\alpha, d, r}\left(R_{V}^{\geq 3}\left(q_{j}^{\prime}\right)\right)^{|\alpha|}
$$

for any $q \in \operatorname{supp} \chi_{j}=B\left(q_{j}, a R_{V}^{\geq 3}\left(q_{j}\right)^{-1}\right)$, where $c_{\alpha, d, r}=\sum_{3 \leq|\beta| \leq r} \beta ! a^{-|\beta|+|\alpha|}$.

As a consequence, if $V$ satisfies Assumption 1, there exists a large constant $\kappa_{1} \geq \kappa_{0}$ so that for all $\kappa \geq \kappa_{1}$

- if $j \in J(\kappa)$

$$
2^{-1}\left|\partial_{q} V_{j}^{2}(q)\right| \leq\left|\partial_{q} V(q)\right| \leq 2\left|\partial_{q} V_{j}^{2}(q)\right| \quad \text { for every } q \in \operatorname{supp} \chi_{j},
$$

- if $j \notin J(\kappa)$

$$
2^{-1}\left|\operatorname{Hess} V_{j}^{2}(q)\right| \leq|\operatorname{Hess} V(q)| \leq 2\left|\operatorname{Hess} V_{j}^{2}(q)\right|
$$

for any $q \in \operatorname{supp} \chi_{j}$ with $|q| \geq C_{2}(\kappa)$ where $C_{2}(\kappa)>0$ is a large constant that depends on $\kappa$. 
Proof. Let $V$ be a polynomial of degree $r$ greater than two. In this proof we are going to need the following equivalence

$$
R_{V}^{\geq 3}(q) \asymp R_{V}^{\geq 3}\left(q^{\prime}\right)
$$

satisfied for all $q, q^{\prime} \in \operatorname{supp} \chi_{j}$ and proved in Lemma A.5. That is there is a constant $C>1$ such that for every $q, q^{\prime} \in \operatorname{supp} \chi_{j}$,

$$
\left(\frac{R_{V}^{\geq 3}(q)}{R_{V}^{\geq 3}\left(q^{\prime}\right)}\right)^{ \pm 1} \leq C
$$

Assume $\alpha \in \mathbb{N}^{d}$ of length $|\alpha| \in\{1,2\}$. For every $j \in \mathbb{N}$, observe that

$$
\begin{aligned}
\left|\partial_{q}^{\alpha} V(q)-\partial_{q}^{\alpha} V_{j}^{2}(q)\right| & =\left|\sum_{\substack{3 \leq|\beta| \leq r \\
\beta \geq \alpha}} \frac{\beta !}{(\beta-\alpha) !} \partial_{q}^{\beta} V\left(q_{j}^{\prime}\right)\left(q-q_{j}^{\prime}\right)^{\beta-\alpha}\right| \\
& \leq \sum_{\substack{3 \leq|\beta| \leq r \\
\beta \geq \alpha}} \frac{\beta !}{(\beta-\alpha) !}\left|\partial_{q}^{\beta} V\left(q_{j}^{\prime}\right)\right|\left|q-q_{j}^{\prime}\right|^{|\beta|-|\alpha|},
\end{aligned}
$$

for any $q \in \mathbb{R}^{d}$. Hence regarding the equivalence (2.9), there exists a constant $c_{\alpha, d, r}>0$ (depending as well on the multi-index $\alpha$, the dimesion $d$ and the degree $\mathrm{r}$ of $V$ ) so that

$$
\begin{aligned}
\left|\partial_{q}^{\alpha} V(q)-\partial_{q}^{\alpha} V_{j}^{2}(q)\right| & \leq \sum_{\substack{3 \leq|\beta| \leq r \\
\beta \geq \alpha}} \frac{\beta !}{(\beta-\alpha) !}\left(R_{V}^{\geq 3}\left(q_{j}^{\prime}\right)\right)^{|\beta|}\left(a R_{V}^{\geq 3}\left(q_{j}\right)\right)^{-|\beta|+|\alpha|} \\
& \leq \sum_{\substack{3 \leq|\beta| \leq r \\
\beta \geq \alpha}} \frac{\beta !}{(\beta-\alpha) !} a^{-|\beta|+|\alpha|}\left(R_{V}^{\geq 3}\left(q_{j}^{\prime}\right)\right)^{|\alpha|} \\
& \leq c_{\alpha, d, r}\left(R_{V}^{\geq 3}\left(q_{j}^{\prime}\right)\right)^{|\alpha|}
\end{aligned}
$$

holds for all $q$ in the support of $\chi_{j}$, where $c_{\alpha, d, r}=\sum_{3 \leq|\beta| \leq r} \beta ! a^{-|\beta|+|\alpha|}$.

In the rest of the proof, let the polynomial $V(q)$ satisfies Assumption 1. In vue of (2.10), we get when $|\alpha|=1$

$$
\left|\nabla V(q)-\nabla V_{j}^{2}(q)\right| \leq c_{1, d, r} R_{V}^{\geq 3}\left(q_{j}^{\prime}\right)
$$

for all $j \in \mathbb{N}$ and any $q \in \operatorname{supp} \chi_{j}$, where $c_{1, d, r}=\sum_{3 \leq|\beta| \leq r} \beta ! a^{-|\beta|+1}$. Given $\kappa \geq \kappa_{0}$, assume first that $j \in J(\kappa)$. By virtue of the equivalence (2.9), it results from (2.11)

$$
\left|\nabla V(q)-\nabla V_{j}^{2}(q)\right| \leq c_{1, d, r} C R_{V}^{\geq 3}(q)
$$


for every $q \in \operatorname{supp} \chi_{j}$. Then we obtain

$$
\begin{aligned}
\left|\nabla V(q)-\nabla V_{j}^{2}(q)\right| & \leq \frac{c_{1, d, r} C}{\kappa^{\frac{1}{4}}}|\nabla V(q)|^{\frac{1}{3}} \\
& \leq \frac{c_{1, d, r} C}{\kappa^{\frac{1}{4}}}|\nabla V(q)|
\end{aligned}
$$

for all $q \in \operatorname{supp} \chi_{j}$. For the above second inequality we know that $|\nabla V(q)| \geq 1$ for every $q \in \operatorname{supp} \chi_{j}$, indeed since $j \in J(\kappa)$,

$$
|\nabla V(q)| \geq \kappa^{\frac{3}{4}} \geq \kappa_{0}^{\frac{3}{4}} \geq 1 .
$$

Taking the constant $\kappa_{1} \geq \kappa_{0}$ such that $\frac{c_{1, d, r} C}{\kappa_{1}^{\frac{1}{4}}} \leq \frac{1}{2}$, we get for every $\kappa \geq \kappa_{1}$

$$
|| \nabla V(q)|-| \nabla V_{j}^{2}(q)|| \leq\left|\nabla V(q)-\nabla V_{j}^{2}(q)\right| \leq \frac{1}{2}|\nabla V(q)|,
$$

for any $q \in \operatorname{supp} \chi_{j}$ when $j \in J(\kappa)$. Therefore

$$
\frac{1}{2}\left|\nabla V_{j}^{2}(q)\right| \leq|\nabla V(q)| \leq \frac{3}{2}\left|\nabla V_{j}^{2}(q)\right|
$$

holds for all $q \in \operatorname{supp} \chi_{j}$ when $j \in J(\kappa)$.

On the other hand when $|\alpha|=2$, by (2.10) and (2.9) there is a constant $c_{2, d, r}>0$ so that for all $j \in \mathbb{N}$

$$
\left|\partial_{q}^{\alpha} V(q)-\partial_{q}^{\alpha} V_{j}^{2}(q)\right| \leq c_{2, d, r} C^{2} R_{V}^{\geq 3}(q)^{2} .
$$

holds for every $q \in \operatorname{supp} \chi_{j}$, where $c_{2, d, r}=\sum_{3 \leq|\beta| \leq r} \beta ! a^{-|\beta|+2}$. Given $\kappa \geq \kappa_{0}$ assume now $j \notin J(\kappa)$. Using the fact that $R_{V}^{\geq 3}(q) \geq R_{V}^{=r}(0)$ for every $q \in \mathbb{R}^{d}$, we derive from (2.14) that

$$
\left|\partial_{q}^{\alpha} V(q)-\partial_{q}^{\alpha} V_{j}^{2}(q)\right| \leq c_{2, d, r} C^{2} \frac{R_{V}^{23}(q)^{4}}{R_{V}^{=r}(0)^{2}}
$$

for all $q \in \operatorname{supp} \chi_{j}$.

Assuming $\kappa \geq \kappa_{0}$ and $j \notin J(\kappa)$, we obtain using the previous inequality and applying Lemma B.6

$$
\left|\sum_{|\alpha|=2}\right| \partial_{q}^{\alpha} V(q)\left|-\sum_{|\alpha|=2}\right| \partial_{q}^{\alpha} V_{j}^{2}(q)|| \leq \sum_{|\alpha|=2}\left|\partial_{q}^{\alpha} V(q)-\partial_{q}^{\alpha} V_{j}^{2}(q)\right| \leq \frac{1}{2}|\operatorname{Hess} V(q)|,
$$

for any $q \in \operatorname{supp} \chi_{j}$ with $|q| \geq C_{2}(\kappa)$ where $C_{2}(\kappa)$ is a strictly positive large constant depending on $\kappa$. In other words,

$$
\frac{1}{2}\left|\operatorname{Hess} V_{j}^{2}(q)\right| \leq|\operatorname{Hess} V(q)| \leq \frac{3}{2}\left|\operatorname{Hess} V_{j}^{2}(q)\right|
$$

holds for all $q \in \operatorname{supp} \chi_{j}$ with $|q| \geq C_{2}(\kappa)$ and $j \notin J(\kappa)$. 
Lemma 2.4. Given two positive operators $A$ and $B$ such that

$$
\|u\|^{2}<\langle u, A u\rangle \leq\langle u, B u\rangle
$$

for all $u \in \mathcal{D}$ where $\mathcal{D}$ is dense in $D\left(A^{1 / 2}\right)$, one has

$$
\left\langle u, \frac{A^{\alpha_{0}}}{\left(\log \left(A^{\alpha_{0} / 2}\right)\right)^{k}} u\right\rangle \leq\left\langle u, \frac{B^{\alpha_{0}}}{\left(\log \left(B^{\alpha_{0} / 2}\right)\right)^{k}} u\right\rangle,
$$

for all $u \in \mathcal{D}$, any $\alpha_{0} \in[0,1]$ and every natural number $k$.

Proof. Assume that $A, B$ are two positive operators so that

$$
\|u\|^{2}<\langle u, A u\rangle \leq\langle u, B u\rangle,
$$

holds for all $u \in \mathcal{D}$. Referring to [Sim] (see Proposition 6.7 and Example 6.8), for any positive operator $C$ and every $\alpha \in(0,1)$ we can write

$$
C^{\alpha}=\frac{2 \sin (\pi \alpha)}{\pi} \int_{0}^{+\infty} w^{\alpha-1}(C+w)^{-1} C d w .
$$

From (2.16) and (2.17)

$$
\|u\|^{2}<\left\langle u, A^{\alpha} u\right\rangle \leq\left\langle u, B^{\alpha} u\right\rangle,
$$

for any $u \in \mathcal{D}$ and every $\alpha \in[0,1]$.

Furthermore, for any positive operator $C$ with domain $D(C)$ we define its logarithm for all $u \in D(C)$ by

$$
\langle u, \log (C) u\rangle=\lim _{\alpha \rightarrow 0^{+}}\left\langle u, \frac{C^{\alpha}-1}{\alpha} u\right\rangle,
$$

where the operator $C^{\alpha}$ is given in (2.17).

Using (2.16) and (2.19)

$$
\|u\|^{2}<\langle u, \log (A) u\rangle \leq\langle u, \log (B) u\rangle,
$$

holds for all $u \in \mathcal{D}$. Integrating (2.18) with respect to $\alpha$ over $\left[0, \alpha_{0}\right]$ where $\alpha_{0} \in[0,1]$ we get

$$
\left\langle u, \frac{1}{\log (A)}\left(A^{\alpha_{0}}-I\right) u\right\rangle \leq\left\langle u, \frac{1}{\log (B)}\left(B^{\alpha_{0}}-I\right) u\right\rangle .
$$

Furthermore by $(2.20)$

$$
\left\langle u, \frac{1}{\log (B)} u\right\rangle \leq\left\langle u, \frac{1}{\log (A)} u\right\rangle<\|u\|^{2} .
$$


Therefore from (2.21) and (2.22)

$$
\left\langle u, \frac{A^{\alpha_{0}}}{\log (A)} u\right\rangle \leq\left\langle u, \frac{B^{\alpha_{0}}}{\log (B)} u\right\rangle .
$$

holds for any $\alpha_{0} \in[0,1]$. Then by induction on $k \in \mathbb{N}$, we obtain

$$
\left\langle u, \frac{A^{\alpha_{0}}}{(\log (A))^{k}} u\right\rangle \leq\left\langle u, \frac{B^{\alpha_{0}}}{(\log (B))^{k}} u\right\rangle
$$

for all $\alpha_{0} \in[0,1]$ and every natural number $k$. Or equivalently

$$
\left\langle u, \frac{A^{\alpha_{0}}}{\left(\log \left(A^{\alpha_{0} / 2}\right)\right)^{k}} u\right\rangle \leq\left\langle u, \frac{B^{\alpha_{0}}}{\left(\log \left(B^{\alpha_{0} / 2}\right)\right)^{k}} u\right\rangle,
$$

for every $\alpha_{0} \in[0,1], k \in \mathbb{N}$.

Lemma 2.5. Assume $V(q)$ a polynomial of degree greater than two. Let $\sum_{j \in \mathbb{N}} \chi_{j}^{2}(q)$ be a locally finite partition of unity defined as in (2.1).

There is a constant $c>0$ such that

$$
\left\langle u,\left(1+D_{q}^{2}+R_{V}^{\geq 3}(q)^{4}\right)^{\alpha} u\right\rangle \leq c \sum_{j \in \mathbb{N}}\left\langle u, \chi_{j}\left(1+D_{q}^{2}+R_{V}^{\geq 3}\left(q_{j}^{\prime}\right)^{4}\right)^{\alpha} \chi_{j} u\right\rangle
$$

is valid for all $u \in \mathcal{C}_{0}^{\infty}\left(\mathbb{R}^{2 d}\right)$ and any $\alpha \in[0,1]$.

As a consequence, there exists a constant $c>0$ so that

$$
\sum_{j \in \mathbb{N}}\left\|L\left(\left(1+D_{q}^{2}+R_{V}^{\geq 3}\left(q_{j}^{\prime}\right)^{4}\right)^{\frac{1}{3}}\right) \chi_{j} u\right\|^{2} \geq \frac{1}{c}\left\|L\left(\left(1+D_{q}^{2}+R_{V}^{\geq 3}(q)^{4}\right)^{\frac{1}{3}}\right) u\right\|^{2},
$$

holds for all $u \in \mathcal{C}_{0}^{\infty}\left(\mathbb{R}^{2 d}\right)$, where $L(s)=\frac{s+1}{\log (s+1)}$ for all $s \geq 1$.

Proof. We first set $E_{0}=L^{2}\left(\mathbb{R}^{2 d}\right)$ and $E_{1}=\left\{u \in L^{2}\left(\mathbb{R}^{2 d}\right),\left\langle u,\left(1-\Delta_{q}+R_{V}^{\geq 3}(q)^{4}\right) u\right\rangle<+\infty\right\}$ endowed respectively with the norms $\|\cdot\|_{E_{0}}=\|\cdot\|_{L^{2}\left(\mathbb{R}^{2 d}\right)}$ and $\|\cdot\|_{E_{1}}$ defined as follows for all $u \in L^{2}\left(\mathbb{R}^{2 d}\right)$

$$
\begin{aligned}
\|u\|_{E_{1}}^{2} & =\|u\|_{L^{2}\left(\mathbb{R}^{2 d}\right)}^{2}+\left\|D_{q} u\right\|_{L^{2}\left(\mathbb{R}^{2 d}\right)}^{2}+\left\|R_{V}^{\geq 3}(q)^{2} u\right\|_{L^{2}\left(\mathbb{R}^{2 d}\right)}^{2} \\
& =\left\|\left(1-\Delta_{q}+R_{V}^{\geq 3}(q)^{4}\right)^{1 / 2} u\right\|_{L^{2}\left(\mathbb{R}^{2 d}\right)}^{2} .
\end{aligned}
$$

By Simader theorem (which states that if $W \in \mathcal{C}^{\infty}\left(\mathbb{R}^{d}\right)$ and $-\Delta+W(x)$ is a symetric non negative operator on $\mathcal{C}_{0}^{\infty}\left(\mathbb{R}^{d}\right)$ then $-\Delta+W(x)$ is essentially self adjoint on $\mathcal{C}_{0}^{\infty}\left(\mathbb{R}^{d}\right)$ ), the operator $1-\Delta_{q}+R_{V}^{\geq 3}(q)^{4}$ is essentially self adjoint on $\mathcal{C}_{0}^{\infty}\left(\mathbb{R}^{2 d}\right)$ and hence $E_{1}$ corresponds to the spectrally defined subspace of $L^{2}\left(\mathbb{R}^{2 d}\right)$. 
Given a partition of unity as in (2.1), define the linear map

$$
T: E_{0} \rightarrow\left(L^{2}\left(\mathbb{R}^{2 d}\right)\right)^{\mathbb{N}}, u \mapsto\left(u_{j}\right)_{j \in \mathbb{N}}=\left(\chi_{j} u\right)_{j \in \mathbb{N}}
$$

and denote $F_{0}:=\operatorname{Im} T$. Notice that $T: E_{0} \rightarrow F_{0}$ is a unitary isometry. Indeed for all $u \in E_{0}$,

$$
\|T u\|_{F_{0}}^{2}=\sum_{j \in \mathbb{N}}\left\|\chi_{j} u\right\|_{L^{2}}^{2}=\|u\|_{L^{2}}^{2}=\|u\|_{E_{0}}^{2},
$$

further the inverse map of $\mathrm{T}$ is well defined by

$$
T^{-1}: F_{0} \rightarrow E_{0},\left(u_{j}\right)_{j \in \mathbb{N}} \mapsto u=\sum_{j \in \mathbb{N}} \chi_{j} u_{j}
$$

Now introduce the set

$$
F_{1}=\left\{\left(u_{j}\right)_{j \in \mathbb{N}} \in F_{0}, \sum_{j \in \mathbb{N}}\left\langle u_{j},\left(1-\Delta_{q}+R_{V}^{\geq 3}\left(q_{j}^{\prime}\right)^{4}\right) u_{j}\right\rangle<+\infty\right\},
$$

with its associated norm defined for all $\left(u_{j}\right)_{j \in \mathbb{N}} \in F_{1}$ by

$$
\begin{aligned}
\left\|\left(u_{j}\right)_{j \in \mathbb{N}}\right\|_{F_{1}}^{2} & =\sum_{j \in \mathbb{N}}\left(\left\|u_{j}\right\|_{L^{2}\left(\mathbb{R}^{2 d}\right)}^{2}+\left\|D_{q} u_{j}\right\|_{L^{2}\left(\mathbb{R}^{2 d}\right)}^{2}+\left\|R_{V}^{\geq 3}\left(q_{j}^{\prime}\right)^{2} u_{j}\right\|_{L^{2}\left(\mathbb{R}^{2 d}\right)}^{2}\right) \\
& =\sum_{j \in \mathbb{N}}\left\|\left(1-\Delta_{q}+R_{V}^{\geq 3}\left(q_{j}^{\prime}\right)^{4}\right)^{1 / 2} u_{j}\right\|_{L^{2}\left(\mathbb{R}^{2 d}\right)}^{2} .
\end{aligned}
$$

Assume $u \in E_{0}$. For all $j \in \mathbb{N}$, let $q_{j}^{\prime} \in \operatorname{supp} \chi_{j}$. Observe that

$$
\begin{aligned}
\left|\|T u\|_{F_{1}}^{2}-\|u\|_{E_{1}}^{2}\right| & =\left|\sum_{j \in \mathbb{N}}\left\langle u_{j},\left(1-\Delta_{q}+R_{V}^{\geq 3}\left(q_{j}^{\prime}\right)^{4}\right) u_{j}\right\rangle-\left\langle u,\left(1-\Delta_{q}+R_{V}^{\geq 3}(q)^{4}\right) u\right\rangle\right| \\
& =\left|\sum_{j \in \mathbb{N}}\left\langle u_{j},-\Delta_{q} u_{j}\right\rangle-\left\langle u,-\Delta_{q} u\right\rangle+\sum_{j \in \mathbb{N}}\left\langle u_{j},\left(R_{V}^{\geq 3}\left(q_{j}^{\prime}\right)^{4}-R_{V}^{\geq 3}(q)^{4}\right) u_{j}\right\rangle\right| \\
& \leq\left|\sum_{j \in \mathbb{N}}\left\langle u_{j},-\Delta_{q} u_{j}\right\rangle-\left\langle u,-\Delta_{q} u\right\rangle\right|+\sum_{j \in \mathbb{N}}\left\langle u_{j},\left|R_{V}^{\geq 3}\left(q_{j}^{\prime}\right)^{4}-R_{V}^{\geq 3}(q)^{4}\right| u_{j}\right\rangle .
\end{aligned}
$$

Since we are dealing with cutoff functions satisfying $\sum_{j \in \mathbb{N}}\left|\nabla \chi_{j}\right|^{2} \leq c R_{V}^{\geq 3}(q)^{2}$ and owning to the equivalence $R_{V}^{\geq 3}(q) \asymp R_{V}^{\geq 3}\left(q_{j}^{\prime}\right)$ for all $q \in \operatorname{supp} \chi_{j}$, it follows from $(2.26)$

$$
\left|\|T u\|_{F_{1}}^{2}-\|u\|_{E_{1}}^{2}\right| \leq c_{1} \sum_{j \in \mathbb{N}}\left\langle u_{j}, R_{V}^{\geq 3}\left(q_{j}^{\prime}\right)^{4} u_{j}\right\rangle \leq c_{1}\|T u\|_{F_{1}}^{2}
$$

and

$$
\left|\|T u\|_{F_{1}}^{2}-\|u\|_{E_{1}}^{2}\right| \leq c_{1}^{\prime}\left\langle u, R_{V}^{\geq 3}(q)^{4} u\right\rangle \leq c_{1}^{\prime}\|u\|_{E_{1}}^{2}
$$


where $c_{1}, c_{1}^{\prime}$ are two strictly positive constants. As a result

$$
\frac{1}{\sqrt{\left(c_{1}+1\right)}}\|u\|_{E_{1}} \leq\|T u\|_{F_{1}} \leq \sqrt{\left(c_{1}^{\prime}+1\right)}\|u\|_{E_{1}} .
$$

In view of (2.25) and (2.27), we conclude by interpolation that for all $\alpha \in[0,1]$

$$
T: E_{\alpha} \rightarrow F_{\alpha}
$$

verifies $\|T\|_{\mathcal{L}\left(E_{\alpha}, F_{\alpha}\right)} \leq c^{\alpha}$ and $\left\|T^{-1}\right\|_{\mathcal{L}\left(F_{\alpha}, E_{\alpha}\right)} \leq c^{\alpha}$, where $E_{\alpha}$ and $F_{\alpha}$ are two interpolated spaces endowed respectively with the norms

$$
\|u\|_{E_{\alpha}}=\left\|\left(1-\Delta_{q}+R_{V}^{\geq 3}(q)^{4}\right)^{\alpha / 2} u\right\|_{L^{2}\left(\mathbb{R}^{2 d}\right)},
$$

and

$$
\left\|\left(v_{j}\right)_{j \in \mathbb{N}}\right\|_{F_{\alpha}}=\sum_{j \in \mathbb{N}}\left\|\left(1-\Delta_{q}+R_{V}^{\geq 3}\left(q_{j}^{\prime}\right)^{4}\right)^{\alpha / 2} u_{j}\right\|_{L^{2}\left(\mathbb{R}^{2 d}\right)}
$$

Hence there is a constant $c>0$ so that

$$
\left\langle u,\left(1+D_{q}^{2}+R_{V}^{\geq 3}(q)^{4}\right)^{\alpha} u\right\rangle \leq c \sum_{j \in \mathbb{N}}\left\langle u, \chi_{j}\left(1+D_{q}^{2}+R_{V}^{\geq 3}\left(q_{j}^{\prime}\right)^{4}\right)^{\alpha} \chi_{j} u\right\rangle,
$$

holds for all $u \in \mathcal{C}_{0}^{\infty}\left(\mathbb{R}^{2 d}\right)$ and any $\alpha \in[0,1]$. In order to prove (2.24), repeat the same process as in Lemma 2.4. Starting with

$$
\|u\|^{2}<\left\langle u,\left(1+D_{q}^{2}+R_{V}^{\geq 3}(q)^{4}\right)^{\alpha} u\right\rangle \leq c \sum_{j \in \mathbb{N}}\left\langle u, \chi_{j}\left(1+D_{q}^{2}+R_{V}^{\geq 3}\left(q_{j}^{\prime}\right)^{4}\right)^{\alpha} \chi_{j} u\right\rangle
$$

for all $u \in \mathcal{C}_{0}^{\infty}\left(\mathbb{R}^{2 d}\right)$ and any $\alpha \in[0,1]$, remark that when integrating over $\alpha \in\left[0, \frac{2}{3}\right]$ we can interchange the sum and the integral in the left hand side of (2.29) since the partition of unity is locally finite.

This completes the proof.

\section{Proof of Theorem 1.1}

In this section we present the proof of Theorem 1.1. In the sequel for a given polynomial $V(q)$ with degree $\mathrm{r}$ greater than two, we always use a locally finite partition of unity

$$
\sum_{j \in \mathbb{N}} \chi_{j}^{2}(q)=\sum_{j \in \mathbb{N}} \widetilde{\chi}_{j}^{2}\left(R_{V}^{\geq 3}\left(q_{j}\right)^{-1}\left(q-q_{j}\right)\right)=1,
$$

where

$$
\operatorname{supp} \widetilde{\chi}_{j} \subset B\left(q_{j}, a\right) \text { and } \tilde{\chi}_{j} \equiv 1 \text { in } B\left(q_{j}, b\right)
$$

for some $q_{j} \in \mathbb{R}^{d}$ with $0<b<a$ independent of the natural numbers $j$, defined more specifically as in Lemma A.7 with $n=3$. 
Proof. Let $V(q)$ be a polynomial with degree larger than two that satisfies Assumption 1. Assume $u \in \mathcal{C}_{0}^{\infty}\left(\mathbb{R}^{2 d}\right)$. In the whole proof we denote $u_{j}=\chi_{j} u$ for all natural number $j$.

From Lemma 2.1 we get

$$
\left\|K_{V} u\right\|_{L^{2}\left(\mathbb{R}^{2 d}\right)}^{2} \geq \sum_{j \in \mathbb{N}}\left\|K_{V} u_{j}\right\|_{L^{2}\left(\mathbb{R}^{2 d}\right)}^{2}-c_{d} R_{V}^{\geq 3}\left(q_{j}\right)^{2}\left\|p u_{j}\right\|_{L^{2}\left(\mathbb{R}^{2 d}\right)}^{2} .
$$

Given $\kappa \geq \kappa_{0}$, set

$$
J(\kappa)=\left\{j \in \mathbb{N} \text {, such that } \operatorname{supp} \chi_{j} \subset \Sigma(\kappa)\right\} .
$$

For all index $j \in \mathbb{N}$, let $V_{j}^{2}$ be the polynomial of degree less than three given by

$$
V_{j}^{2}(q)=\sum_{0 \leq|\alpha| \leq 2} \frac{\partial_{q}^{\alpha} V\left(q_{j}^{\prime}\right)}{\alpha !}\left(q-q_{j}^{\prime}\right)^{\alpha}
$$

where

$$
\begin{cases}q_{j}^{\prime}=q_{j} & \text { if } j \in J(\kappa) \\ q_{j}^{\prime} \in\left(\operatorname{supp} \chi_{j}\right) \cap\left(\mathbb{R}^{d} \backslash \Sigma(\kappa)\right) & \text { else. }\end{cases}
$$

We associate with each polynomial $V_{j}^{2}$ the Kramers-Fokker-Planck operator $K_{V_{j}^{2}}$. Observe that using the parallelogram law $2\left(\|x\|^{2}+\|y\|^{2}\right)-\|x+y\|^{2}=\|x-y\|^{2} \geq 0$,

$$
\begin{aligned}
\sum_{j \in \mathbb{N}}\left\|K_{V} u_{j}\right\|_{L^{2}\left(\mathbb{R}^{2 d}\right)}^{2} & =\sum_{j \in \mathbb{N}}\left\|K_{V_{j}^{2}} u_{j}+\left(K_{V}-K_{V_{j}^{2}}\right) u_{j}\right\|_{L^{2}\left(\mathbb{R}^{2 d}\right)}^{2} \\
& \geq \frac{1}{2} \sum_{j \in \mathbb{N}}\left\|K_{V_{j}^{2}} u_{j}\right\|_{L^{2}\left(\mathbb{R}^{2 d}\right)}^{2}-\left\|\left(\nabla V(q)-\nabla V_{j}^{2}(q)\right) \partial_{p} u_{j}\right\|_{L^{2}\left(\mathbb{R}^{2 d}\right)}^{2}
\end{aligned}
$$

On the other hand, by (2.5) in Lemma 2.3

$$
\sum_{j \in \mathbb{N}}\left\|\left(\nabla V(q)-\nabla V_{j}^{2}(q)\right) \partial_{p} u_{j}\right\|_{L^{2}\left(\mathbb{R}^{2 d}\right)}^{2} \leq c_{1, d, r} \sum_{j \in \mathbb{N}} R_{V}^{23}\left(q_{j}^{\prime}\right)^{2}\left\|\partial_{p} u_{j}\right\|_{L^{2}\left(\mathbb{R}^{2 d}\right)}^{2} .
$$

Combining (3.1), (3.2) and (3.3) we get immediately

$$
\left\|K_{V} u\right\|_{L^{2}\left(\mathbb{R}^{2 d}\right)}^{2} \geq \frac{1}{2} \sum_{j \in \mathbb{N}}\left\|K_{V_{j}^{2}} u_{j}\right\|_{L^{2}\left(\mathbb{R}^{2 d}\right)}^{2}-c_{1, d, r} R_{V}^{\geq 3}\left(q_{j}^{\prime}\right)^{2}\left\|\partial_{p} u_{j}\right\|_{L^{2}\left(\mathbb{R}^{2 d}\right)}^{2}-c_{d} R_{V}^{\geq 3}\left(q_{j}\right)^{2}\left\|p u_{j}\right\|_{L^{2}\left(\mathbb{R}^{2 d}\right)}^{2}
$$

Therefore, making use of the equivalence $(A .5)$, it follows

$$
\left\|K_{V} u\right\|_{L^{2}\left(\mathbb{R}^{2 d}\right)}^{2} \geq \frac{1}{2} \sum_{j \in \mathbb{N}}\left\|K_{V_{j}^{2}} u_{j}\right\|_{L^{2}\left(\mathbb{R}^{2 d}\right)}^{2}-c_{d, r}^{\prime} R_{V}^{\geq 3}\left(q_{j}^{\prime}\right)^{2}\left\langle u_{j}, O_{p} u_{j}\right\rangle_{L^{2}\left(\mathbb{R}^{2 d}\right)},
$$


where $c_{d, r}^{\prime}=2\left(c_{1, d, r}^{2}+c_{d}\right)$.

Using respectively the Cauchy Schwarz inequality then the Cauchy inequality with epsilon (for any real numbers $a, b$ and all $\epsilon>0, a b \leq \epsilon a^{2}+\frac{1}{4 \epsilon} b^{2}$ ),

$$
\begin{aligned}
c_{d, r}^{\prime} R_{V}^{\geq 3}\left(q_{j}^{\prime}\right)^{2}\left\langle u_{j}, O_{p} u_{j}\right\rangle & =c_{d, r}^{\prime} R_{V}^{\geq 3}\left(q_{j}^{\prime}\right)^{2} \operatorname{Re}\left\langle u_{j}, K_{V_{j}^{2}} u_{j}\right\rangle \\
& \leq c_{d, r}^{\prime} R_{V}^{\geq 3}\left(q_{j}^{\prime}\right)^{2}\left\|u_{j}\right\| \cdot\left\|K_{V_{j}^{2}} u_{j}\right\| \\
& \leq\left(c_{d, r}^{\prime} R_{V}^{\geq 3}\left(q_{j}^{\prime}\right)^{2}\right)^{2}\left\|u_{j}\right\|^{2}+\frac{1}{4}\left\|K_{V_{j}^{2}} u_{j}\right\|^{2} .
\end{aligned}
$$

Putting the above estimate and (3.4) together we obtain

$$
\left\|K_{V} u\right\|^{2} \geq \sum_{j \in \mathbb{N}} \frac{1}{4}\left\|K_{V_{j}^{2}} u_{j}\right\|^{2}-\left(c_{d, r}^{\prime}\right)^{2} R_{V}^{\geq 3}\left(q_{j}^{\prime}\right)^{4}\left\|u_{j}\right\|^{2}
$$

From now on assume $\kappa \geq \kappa_{1}$, where $\kappa_{1} \geq \kappa_{0}$ is introduced in Lemma 2.3. Remember as well that the constants $C_{1}, C_{2}(\kappa)$ are given respectively in Assumption 1 (see (1.8)) and Lemma 2.3 (see (2.7)). By introducing $C(\kappa) \geq \max \left(C_{1}, C_{2}(\kappa)\right)$, which will be fixed later, we set for each $\kappa$,

$$
I(\kappa)=\left\{j \in \mathbb{N}, \text { such that } \operatorname{supp} \chi_{j} \subset\left\{q \in \mathbb{R}^{d},|q| \geq C(\kappa)\right\}\right\} .
$$

The rest of the proof is divided into three steps. The first one is devoted to the control of the terms in the the left hand side of (3.5) for which $j \in I(\kappa)$ for some large $\kappa \geq \kappa_{0}$ to be chosen. At the end of the first step the constants $\kappa>\kappa_{1}$ and $C(\kappa) \geq \max \left(C_{1}, C_{2}(\kappa)\right)$ will be fixed. So on, the second step is concerned with the remaining terms for which the support of the cutoff functions $\chi_{j}$ are included in some closed ball $B\left(0, C^{\prime}(\kappa)\right)$. We finally sum up all the terms and refer to Lemma 2.5 after some elementary optimization trick in the last step.

Step 1, $\mathbf{j} \in \mathbf{I}(\kappa), \kappa \geq \kappa_{\mathbf{1}}$ to be fixed: As proved in [BNV], there is a constant $c>0$ such that for all $j \in I(\kappa)$

$$
\left\|K_{V_{j}^{2}} u_{j}\right\|^{2}+A_{V_{j}^{2}}\left\|u_{j}\right\|^{2} \geq c\left(\left\|O_{p} u_{j}\right\|^{2}+\left\|\left\langle\partial_{q} V_{j}^{2}(q)\right\rangle^{2 / 3} u_{j}\right\|^{2}+\left\|\left\langle D_{q}\right\rangle^{2 / 3} u_{j}\right\|\right),
$$

where

$$
\begin{aligned}
A_{V_{j}^{2}} & =\max \left\{\left(1+\operatorname{Tr}_{+, V_{j}^{2}}\right)^{2 / 3}, 1+\operatorname{Tr}_{-, V_{j}^{2}}\right\} \\
& =\max \left\{\left(1+\operatorname{Tr}_{+, V}\left(q_{j}^{\prime}\right)\right)^{2 / 3}, 1+\operatorname{Tr}_{-, V}\left(q_{j}^{\prime}\right)\right\} .
\end{aligned}
$$

Hence there is a constant $C>0$ so that

$$
\begin{aligned}
\left\|K_{V_{j}^{2}} u_{j}\right\|^{2}+(1+10 C) t_{j}^{4}\left\|u_{j}\right\|^{2} \geq C\left(\left\|O_{p} u_{j}\right\|^{2}\right. & +\left\|\left\langle\partial_{q} V_{j}^{2}(q)\right\rangle^{2 / 3} u_{j}\right\|^{2} \\
& \left.+\left\|\left\langle D_{q}\right\rangle^{2 / 3} u_{j}\right\|+10 C t_{j}^{4}\left\|u_{j}\right\|^{2}\right)
\end{aligned}
$$


where we use the notation $t_{j}=2\left\langle\operatorname{Hess} V\left(q_{j}^{\prime}\right)\right\rangle^{1 / 4}$ in the whole of the proof.

Recall that as mentioned in $[\mathrm{BNV}]$, the constant $c$ in (3.6) does not depend on the polynomial $V_{j}^{2}$ and then so is the constant $C$ in (3.7).

Now for all index $j \in I(\kappa)$ we distinguish two cases: either $j \in J(\kappa)$ or $j \notin J(\kappa)$.

Case 1. Assume $j \in J(\kappa)$. Then taking into account the inequality (2.6) in Lemma 2.3 and using the estimate (3.7) we obtain

$$
\begin{aligned}
\left\|K_{V_{j}^{2}} u_{j}\right\|^{2}+(1+10 C) t_{j}^{4}\left\|u_{j}\right\|^{2} \geq C\left(\left\|O_{p} u_{j}\right\|^{2}\right. & +\left\|\left\langle\partial_{q} V(q)\right\rangle^{2 / 3} u_{j}\right\|^{2} \\
& \left.+\left\|\left\langle D_{q}\right\rangle^{2 / 3} u_{j}\right\|+10 t_{j}^{4}\left\|u_{j}\right\|^{2}\right),
\end{aligned}
$$

Furthermore, since for all index $j \in \mathbb{N}$ the quantity $R_{V}^{\geq 2}\left(q_{j}^{\prime}\right)^{2}$ is always greater than $\mid$ Hess $V\left(q_{j}^{\prime}\right) \mid$, there exists a constant $c_{d}>0$ so that for every $j \in J(\kappa)$,

$$
t_{j}^{4}=16\left\langle\operatorname{Hess} V\left(q_{j}^{\prime}\right)\right\rangle \leq c_{d}\left\langle R_{V}^{\geq 2}\left(q_{j}^{\prime}\right)^{2}\right\rangle .
$$

Using the fact that the metric $R_{V}^{\geq 2}(q) d q^{2}$ is $R_{V}^{\geq 3}(q) d q^{2}$ slow (see Definition (A.2) and Lemma A.5), it follows

$$
t_{j}^{4} \leq c_{d}\left\langle R_{V}^{\geq 2}(q)^{2}\right\rangle
$$

for every $q \in \operatorname{supp} \chi_{j}$. Hence there is a constant $c_{d}^{\prime}>0$ (depending on the dimension $d$ ) such that

$$
\begin{aligned}
t_{j}^{4} & \leq c_{d}\left\langle\left(\sum_{|\alpha|=2}\left|\partial_{q}^{\alpha} V(q)\right|^{\frac{1}{|\alpha|}}+R_{V}^{\geq 3}(q)\right)^{2}\right\rangle \\
& \leq 3 c_{d}\left\langle\left(\sum_{|\alpha|=2}\left|\partial_{q}^{\alpha} V(q)\right|^{\frac{1}{\alpha \mid}}\right)^{2}+R_{V}^{\geq 3}(q)^{2}\right\rangle \\
& \leq c_{d}^{\prime}\left\langle|\operatorname{Hess} V(q)|+R_{V}^{\geq 3}(q)^{2}\right\rangle
\end{aligned}
$$

holds for any $q \in \operatorname{supp} \chi_{j}$. Or since for every $q \in \mathbb{R}^{d}$ on has $R_{V}^{\geq 3}(q) \geq R_{V}^{=r}(0)$, we derive from the previous estimate that for any $q \in \operatorname{supp} \chi_{j}$,

$$
\begin{aligned}
t_{j}^{4} & \leq c_{d}^{\prime}\left\langle|\operatorname{Hess} V(q)|+\frac{R_{V}^{\geq 3}(q)^{4}}{R_{V}^{=r}(0)^{2}}\right\rangle \\
& \leq \frac{c^{\prime \prime} d}{\kappa} \max \left(1, R_{V}^{=r}(0)^{-2}\right)\left\langle\partial_{q} V(q)\right\rangle^{\frac{4}{3}} .
\end{aligned}
$$

Collecting the estimates (3.8) and (3.9), we get for $\kappa \geq \kappa_{1}$

$$
\begin{aligned}
\left\|K_{V_{j}^{2}} u_{j}\right\|^{2}+(1+10 C) \frac{c^{\prime \prime} d}{\kappa} & \max \left(1, R_{V}^{=r}(0)^{-2}\right)\left\|\left\langle\partial_{q} V(q)\right\rangle^{\frac{2}{3}} u_{j}\right\|^{2} \\
& \geq C\left(\left\|O_{p} u_{j}\right\|^{2}+\left\|\left\langle\partial_{q} V(q)\right\rangle^{2 / 3} u_{j}\right\|^{2}+\left\|\left\langle D_{q}\right\rangle^{2 / 3} u_{j}\right\|^{2}+10 t_{j}^{4}\left\|u_{j}\right\|^{2}\right) .
\end{aligned}
$$


Choosing $\kappa_{2} \geq \kappa_{1}$ so that

$$
\frac{C}{2} \geq(1+10 C) \frac{c^{\prime \prime} d}{\kappa_{2}} \max \left(1, R_{V}^{=r}(0)^{-2}\right),
$$

the following inequality

$$
\left\|K_{V_{j}^{2}} u_{j}\right\|^{2} \geq C\left(\left\|O_{p} u_{j}\right\|^{2}+\frac{1}{2}\left\|\left\langle\partial_{q} V(q)\right\rangle^{2 / 3} u_{j}\right\|^{2}+\left\|\left\langle D_{q}\right\rangle^{2 / 3} u_{j}\right\|^{2}+10 t_{j}^{4}\left\|u_{j}\right\|^{2}\right),
$$

holds for all $j \in J(\kappa)$ with $\kappa \geq \kappa_{2}$.

Or since $j \in J(\kappa)$, there is a constant $c_{1}>0$ so that

$$
\frac{1}{8}\left\langle\partial_{q} V(q)\right\rangle^{\frac{4}{3}} \geq c_{1}\langle\operatorname{Hess} V(q)\rangle,
$$

holds for all $q \in \operatorname{supp} \chi_{j}$. In addition, using the equivalence (A.5) it follows

$$
\frac{1}{8}\left\langle\partial_{q} V(q)\right\rangle^{\frac{4}{3}} \geq c_{2}\left|\partial_{q} V(q)\right|^{\frac{4}{3}} \geq c_{2} \kappa R_{V}^{\geq 3}(q)^{4} \geq c_{2}^{\prime} \kappa R_{V}^{\geq 3}\left(q_{j}^{\prime}\right)^{4},
$$

for any $q \in \operatorname{supp} \chi_{j}$.

Putting (3.10), (3.11) and (3.12) together,

$$
\begin{aligned}
\left\|K_{V_{j}^{2}} u_{j}\right\|^{2} \geq C\left(\left\|O_{p} u_{j}\right\|^{2}\right. & +\frac{1}{4}\left\|\left\langle\partial_{q} V(q)\right\rangle^{2 / 3} u_{j}\right\|^{2}+\left\|\left\langle D_{q}\right\rangle^{2 / 3} u_{j}\right\|^{2} \\
& \left.+c_{1}\left\|\langle\operatorname{Hess} V(q)\rangle^{1 / 2} u_{j}\right\|^{2}+c_{2}^{\prime} \kappa R_{V}^{\geq 3}\left(q_{j}^{\prime}\right)^{4}\left\|u_{j}\right\|^{2}+10\left\|t_{j}^{2} u_{j}\right\|^{2}\right),
\end{aligned}
$$

holds for all $\kappa \geq \kappa_{2}$.

Case 2. Assume $j \notin J(\kappa)$, with $\kappa \geq \kappa_{2} \geq \kappa_{1} \geq \kappa_{0}$. Hence by Assumption 1 (see (1.8)), one has

$$
\operatorname{Tr}_{-, V}(q) \neq 0 \text { for all } q \in\left(\operatorname{supp} \chi_{j}\right) \cap\left(\mathbb{R}^{d} \backslash \Sigma(\kappa)\right) \text { such that }|q| \geq C_{1} .
$$

In particular, since $\left|q_{j}^{\prime}\right| \geq C(\kappa) \geq C_{1}$,

$$
\operatorname{Tr}_{-, V_{j}^{2}}=\operatorname{Tr}_{-, V}\left(q_{j}^{\prime}\right) \neq 0 .
$$

Then referring again to $[\mathrm{BNV}]$,

$$
\left\|K_{V_{j}^{2}} u_{j}\right\|^{2} \geq c B_{V_{j}^{2}}\left\|u_{j}\right\|^{2}
$$

where

$$
\begin{aligned}
B_{V_{j}^{2}} & =\max \left(\min _{q \in \mathbb{R}^{d}}\left|\nabla V_{j}^{2}(q)\right|^{4 / 3}, \frac{1+\operatorname{Tr}_{-, V_{j}^{2}}}{\log \left(2+\operatorname{Tr}_{-, V_{j}^{2}}\right)^{2}}\right) \\
& =\max \left(\min _{q \in \mathbb{R}^{d}}\left|\nabla V_{j}^{2}(q)\right|^{4 / 3}, \frac{1+\operatorname{Tr}_{-, V}\left(q_{j}^{\prime}\right)}{\log \left(2+\operatorname{Tr}_{-, V}\left(q_{j}^{\prime}\right)\right)^{2}}\right) \neq 0 .
\end{aligned}
$$


Hence we get in particular

$$
\left\|K_{V_{j}^{2}} u_{j}\right\|^{2} \geq \frac{1+\operatorname{Tr}_{-, V}\left(q_{j}^{\prime}\right)}{\log \left(2+\operatorname{Tr}_{-, V}\left(q_{j}^{\prime}\right)\right)^{2}}\left\|u_{j}\right\|^{2} .
$$

Using again the condition (1.8) in Assumption 1, there is a constant $C_{1} \geq 1$ so that

$$
\frac{1}{2} \operatorname{Tr}_{-, V}\left(q_{j}^{\prime}\right) \geq \frac{1}{2 C_{1}} \operatorname{Tr}_{+, V}\left(q_{j}^{\prime}\right),
$$

holds, which in turn implies

$$
\operatorname{Tr}_{-, V}\left(q_{j}^{\prime}\right) \geq \frac{1}{2} \operatorname{Tr}_{-, V}\left(q_{j}^{\prime}\right)+\frac{1}{2 C_{1}} \operatorname{Tr}_{+, V}\left(q_{j}^{\prime}\right) \geq \frac{1}{2 C_{1}}\left(\operatorname{Tr}_{-, V}\left(q_{j}^{\prime}\right)+\operatorname{Tr}_{+, V}\left(q_{j}^{\prime}\right)\right),
$$

Then it follows from (3.14) and (3.15)

$$
\left\|K_{V_{j}^{2}} u_{j}\right\|^{2} \geq c^{\prime}\left\|\frac{\sqrt{1+\left|\operatorname{Hess} V\left(q_{j}^{\prime}\right)\right|}}{\log \left(2+\left|\operatorname{Hess} V\left(q_{j}^{\prime}\right)\right|\right)} u_{j}\right\|^{2} .
$$

By Assumption 1 (see condition (1.9)) and (3.16), applying Lemma B.6, there is $\delta \in(0,1)$ and $\Lambda_{\Sigma}(\varrho), \lim _{\varrho \rightarrow+\infty} \Lambda_{\Sigma(\kappa)}(\varrho)=+\infty$ such that

$$
\begin{aligned}
\frac{1+\left|\operatorname{Hess} V\left(q_{j}^{\prime}\right)\right|}{\log \left(2+\left|\operatorname{Hess} V\left(q_{j}^{\prime}\right)\right|\right)^{2}} & \geq \frac{1}{2^{2(1-\delta)}}\left(1+\left|\operatorname{Hess} V\left(q_{j}^{\prime}\right)\right|\right)^{1-\delta} \\
& \geq \frac{1}{4}\left|\operatorname{Hess} V\left(q_{j}^{\prime}\right)\right|^{1-\delta} \\
& \geq \frac{\Lambda_{\Sigma(\kappa)}\left(\left|q_{j}^{\prime}\right|\right)}{4} R_{V}^{\geq 3}\left(q_{j}^{\prime}\right)^{4} \geq \frac{\Lambda_{\Sigma(\kappa)}(C(\kappa))}{4} R_{V}^{\geq 3}\left(q_{j}^{\prime}\right)^{4} .
\end{aligned}
$$

Therefore we get from the above inequality and (3.16),

$$
\left\|K_{V_{j}^{2}} u_{j}\right\|^{2} \geq \Lambda_{\Sigma(\kappa)}(C(\kappa)) R_{V}^{\geq 3}\left(q_{j}^{\prime}\right)^{4}\left\|u_{j}\right\|^{2} .
$$

Next, remind that $t_{j}=2\left\langle\text { Hess } V\left(q_{j}^{\prime}\right)\right\rangle^{1 / 4}$. By (2.7) in Lemma 2.3, the equivalence

$$
t_{j} \asymp 2\langle\operatorname{Hess} V(q)\rangle^{1 / 4} \text {, }
$$

holds for any $q \in \operatorname{supp} \chi_{j}$ with $|q| \geq C(\kappa) \geq C_{2}(\kappa)$. From (3.7) and (3.18) we see that

$$
\begin{aligned}
\left\|K_{V_{j}^{2}} u_{j}\right\|^{2}+(1+10 C) t_{j}^{4}\left\|u_{j}\right\|^{2} \geq C\left(\left\|O_{p} u_{j}\right\|^{2}\right. & +\left\|\langle\operatorname{Hess} V(q)\rangle^{1 / 2} u_{j}\right\|^{2} \\
& \left.+\left\|\left\langle D_{q}\right\rangle^{2 / 3} u_{j}\right\|+9 C t_{j}^{4}\left\|u_{j}\right\|^{2}\right),
\end{aligned}
$$

Since $j \notin J(\kappa)$

$$
|\operatorname{Hess} V(q)|+R_{V}^{\geq 3}(q)^{4}+1 \geq \frac{1}{\kappa}|\nabla V(q)|^{\frac{4}{3}},
$$


for all $q \in \operatorname{supp} \chi_{j}$. Furthermore, it results by Lemma B.6 that for all $q \in \operatorname{supp} \chi_{j}$ (where $j \notin J(\kappa))$

$$
|\operatorname{Hess} V(q)|+R_{V}^{\geq 3}(q)^{4}+1 \leq \frac{3}{2}|\operatorname{Hess} V(q)| .
$$

From (3.20) and (3.21) we get

$$
|\operatorname{Hess} V(q)| \geq \frac{1}{2 \kappa}|\nabla V(q)|^{\frac{4}{3}} \quad, \quad|\operatorname{Hess} V(q)| \geq \frac{2}{3} \geq \frac{1}{2 \kappa} .
$$

Hence there exists a constant $c^{\prime \prime}>0$ such that

$$
\langle\operatorname{Hess} V(q)\rangle \geq \frac{c^{\prime \prime}}{\kappa}\left\langle\partial_{q} V(q)\right\rangle^{4 / 3}
$$

for any $q \in \operatorname{supp} \chi_{j}$ with $|q| \geq C(\kappa) \geq C_{2}(\kappa)$.

The above inequality combined with (3.19) leads to

$$
\begin{aligned}
\left\|K_{V_{j}^{2}} u_{j}\right\|^{2}+(1+10 C) t_{j}^{4}\left\|u_{j}\right\|^{2} \geq C( & \left\|O_{p} u_{j}\right\|^{2}+\left\|\left\langle D_{q}\right\rangle^{2 / 3} u_{j}\right\|^{2}+9 t_{j}^{4}\left\|u_{j}\right\|^{2} \\
& \left.+\frac{c^{\prime \prime}}{\kappa}\left\|\left\langle\partial_{q} V(q)\right\rangle^{2 / 3} u_{j}\right\|^{2}+\frac{1}{2}\left\|\langle\operatorname{Hess} V(q)\rangle^{1 / 2} u_{j}\right\|^{2}\right),
\end{aligned}
$$

for all $\kappa \geq \kappa_{2}$

Collecting the estimates (3.22) and (3.16) we get

$$
\begin{aligned}
\log \left(t_{j}^{4}\right)^{2}\left\|K_{V_{j}^{2}} u_{j}\right\|^{2} \geq C^{\prime \prime}\left(\left\|O_{p} u_{j}\right\|^{2}\right. & +\left\|\left\langle D_{q}\right\rangle^{2 / 3} u_{j}\right\|^{2}+9 t_{j}^{4}\left\|u_{j}\right\|^{2} \\
& \left.+\frac{c^{\prime \prime}}{\kappa}\left\|\left\langle\partial_{q} V(q)\right\rangle^{2 / 3} u_{j}\right\|^{2}+\frac{1}{2}\left\|\langle\operatorname{Hess} V(q)\rangle^{1 / 2} u_{j}\right\|^{2}\right) .
\end{aligned}
$$

In order to reduce the written expressions we denote

$$
\Lambda_{1, j}=\frac{O_{p}}{\log \left(t_{j}^{4}\right)}, \quad \Lambda_{2, j}=\frac{\langle\operatorname{Hess} V(q)\rangle^{1 / 2}}{\log \left(t_{j}^{4}\right)}, \quad \Lambda_{3, j}=\frac{\left\langle\partial_{q} V(q)\right\rangle^{2 / 3}}{\log \left(t_{j}^{4}\right)}, \quad \Lambda_{4, j}=\frac{t_{j}^{2}}{\log \left(t_{j}^{4}\right)} .
$$

The estimate (3.23) can be rewritten as follows

$$
\left\|K_{V_{j}^{2}} u_{j}\right\|^{2} \geq C^{\prime \prime}\left(\left\|\Lambda_{1, j} u_{j}\right\|^{2}+\frac{1}{2}\left\|\Lambda_{2, j} u_{j}\right\|^{2}+\frac{c^{\prime \prime}}{2 \kappa}\left\|\Lambda_{3, j} u_{j}\right\|^{2}+9\left\|\Lambda_{4, j} u_{j}\right\|^{2}+\left\|\frac{\left\langle D_{q}\right\rangle^{2 / 3}}{\log \left(t_{j}^{4}\right)} u_{j}\right\|^{2}\right) .
$$

Using (3.24) and (3.17) we obtain

$$
\begin{aligned}
\left(1+C^{\prime \prime}\right)\left\|K_{V_{j}^{2}} u_{j}\right\|^{2} \geq C^{\prime \prime}\left(\left\|\Lambda_{1, j} u_{j}\right\|^{2}\right. & +\frac{1}{2}\left\|\Lambda_{2, j} u_{j}\right\|^{2}+\frac{c^{\prime \prime}}{2 \kappa}\left\|\Lambda_{3, j} u_{j}\right\|^{2}+9\left\|\Lambda_{4, j} u_{j}\right\|^{2} \\
& \left.+\left\|\frac{\left\langle D_{q}\right\rangle^{2 / 3}}{\log \left(t_{j}^{4}\right)} u_{j}\right\|^{2}+\Lambda_{\Sigma(\kappa)}(C(\kappa)) R_{V}^{\geq 3}\left(q_{j}^{\prime}\right)^{4}\left\|u_{j}\right\|^{2}\right) .
\end{aligned}
$$


Therefore in both cases, that is for all $j \in I(\kappa)$ where $\kappa \geq \kappa_{2}$

$$
\begin{aligned}
\left\|K_{V_{j}^{2}} u_{j}\right\|^{2} \geq C^{(3)}\left(\left\|\Lambda_{1, j} u_{j}\right\|^{2}\right. & +\left\|\Lambda_{2, j} u_{j}\right\|^{2}+\frac{1}{\kappa}\left\|\Lambda_{3, j} u_{j}\right\|^{2}+\left\|\Lambda_{4, j} u_{j}\right\|^{2} \\
& \left.+\left\|\frac{\left\langle D_{q}\right\rangle^{2 / 3}}{\log \left(t_{j}^{4}\right)} u_{j}\right\|^{2}+\min \left(\kappa, \Lambda_{\Sigma(\kappa)}(C(\kappa))\right) R_{V}^{\geq 3}\left(q_{j}^{\prime}\right)^{4}\left\|u_{j}\right\|^{2}\right) .
\end{aligned}
$$

Due to the elementary inequality $u^{4 / 3}+v^{4} \geq \frac{1}{c_{0}}\left(u^{2}+v^{4}\right)^{2 / 3}$ satisfied for all $u, v \geq 1$, we obtain for all $\kappa \geq \kappa_{2}$

$$
\left\|\frac{\left\langle D_{q}\right\rangle^{2 / 3}}{\log \left(t_{j}^{4}\right)} u_{j}\right\|^{2}+\frac{1}{2} \min \left(\kappa, \Lambda_{\Sigma(\kappa)}(C(\kappa))\right) R_{V}^{\geq 3}\left(q_{j}^{\prime}\right)^{4}\left\|u_{j}\right\|^{2} \geq \frac{1}{c_{0}}\left\|\Lambda_{5, j} u_{j}\right\|^{2},
$$

where

$$
\Lambda_{5, j}=\frac{\left(1+D_{q}^{2}+R_{V}^{\geq 3}\left(q_{j}^{\prime}\right)^{4}\right)^{\frac{1}{3}}}{\log \left(t_{j}^{4}\right)} .
$$

In conclusion, we get by (3.25) and (3.26) for every $j \in I(\kappa)$ with $\kappa \geq \kappa_{2}$

$$
\begin{aligned}
\left\|K_{V_{j}^{2}} u_{j}\right\|^{2} \geq C^{(3)}\left(\left\|\Lambda_{1, j} u_{j}\right\|^{2}+\left\|\Lambda_{2, j} u_{j}\right\|^{2}\right. & +\frac{1}{\kappa}\left\|\Lambda_{3, j} u_{j}\right\|^{2}+\left\|\Lambda_{4, j} u_{j}\right\|^{2} \\
& \left.+\frac{1}{c_{0}}\left\|\Lambda_{5, j} u_{j}\right\|^{2}+\frac{1}{2} \min \left(\kappa, \Lambda_{\Sigma(\kappa)}(C(\kappa))\right) R_{V}^{\geq 3}\left(q_{j}^{\prime}\right)^{4}\left\|u_{j}\right\|^{2}\right) .
\end{aligned}
$$

We now fix the choice firstly of $C(\kappa)$ and secondly of $\kappa$. Because $\lim _{\varrho \rightarrow+\infty} \Lambda_{\Sigma(\kappa)}(\varrho)=+\infty$, we can choose for any $\kappa \geq \kappa_{2}, C(\kappa) \geq \max \left(C_{1}, C_{2}(\kappa)\right)$ such that $\Lambda_{\Sigma(\kappa)}(C(\kappa)) \geq \kappa$. We then choose $\kappa=\kappa_{3} \geq \kappa_{2}$ such that

$$
\frac{C^{(3)}}{8} \min \left(\kappa_{3}, \Lambda_{\Sigma\left(\kappa_{3}\right)}\left(\kappa_{3}\right)\right)=\frac{C^{(3)} \kappa_{3}}{8} \geq\left(c_{d, r}^{\prime}\right)^{2}
$$

where $c_{d, r}^{\prime}$ is the constant in (3.5),

$$
\begin{aligned}
\sum_{j \in I\left(\kappa_{3}\right)} \frac{1}{4}\left\|K_{V_{j}^{2}} u\right\|^{2}-\left(c_{d, r}^{\prime}\right)^{2} R_{V}^{\geq 3}\left(q_{j}^{\prime}\right)^{4}\left\|u_{j}\right\|^{2} \geq \frac{C^{(3)}}{8} & \sum_{j \in I\left(\kappa_{3}\right)}\left(\left\|\Lambda_{1, j} u_{j}\right\|^{2}+\left\|\Lambda_{2, j} u_{j}\right\|^{2}\right. \\
& \left.+\frac{1}{\kappa_{3}}\left\|\Lambda_{3, j} u_{j}\right\|^{2}+\left\|\Lambda_{4, j} u_{j}\right\|^{2}+\frac{1}{c_{0}}\left\|\Lambda_{5, j} u_{j}\right\|^{2}\right) .
\end{aligned}
$$

Step 2, $\mathbf{j} \notin \mathbf{I}\left(\kappa_{\mathbf{3}}\right)$ : The set $\mathbb{N} \backslash I\left(\kappa_{3}\right)$ is now a fixed finite set and we can define

$$
\begin{aligned}
C^{(4)}=\max _{j \in \mathbb{N} \backslash I\left(\kappa_{3}\right)}\left[A_{V_{j}^{2}}+\sup _{q \in \operatorname{supp} \chi_{j}}(\langle\operatorname{Hess} V(q)\rangle+\right. & \left.\left\langle\partial_{q} V(q)\right\rangle^{4 / 3}\right) \\
& \left.+\frac{t_{j}^{4}}{\log \left(t_{j}^{4}\right)^{2}}+\left(1+\left(c_{d, r}^{\prime}\right)^{2}\right)\left(1+R_{V}^{\geq 3}\left(q_{j}^{\prime}\right)\right)^{4}\right] .
\end{aligned}
$$


From the lower bound (1.5) we deduce

$$
\begin{array}{r}
\frac{1}{4}\left\|K_{V_{j}^{2}} u_{j}\right\|+C^{(4)}\left\|u_{j}\right\|^{2}-\left(c_{d, r}^{\prime}\right)^{2} R_{V}^{\geq 3}\left(q_{j}^{\prime}\right)^{4}\left\|u_{j}\right\|^{2} \geq \frac{c}{4}\left[\left\|O_{p} u_{j}\right\|^{2}+\left\|\left\langle D_{q}\right\rangle^{2 / 3} u_{j}\right\|^{2}\right]+\left(1+R_{V}^{\geq 3}\left(q_{j}^{\prime}\right)\right)^{4}\left\|u_{j}\right\|^{2} \\
+\left\|\left\langle\partial_{q} V(q)\right\rangle^{2 / 3} u_{j}\right\|^{2}+\left\|\langle\operatorname{Hess} V(q)\rangle^{1 / 2} u_{j}\right\|^{2}+\left\|\frac{t_{j}^{2}}{\log \left(t_{j}^{4}\right)} u_{j}\right\|^{2}
\end{array}
$$

With the quantities

$$
\begin{aligned}
\Lambda_{1, j}=\frac{O_{p}}{\log \left(t_{j}^{4}\right)}, \quad \Lambda_{2, j} & =\frac{\langle\operatorname{Hess} V(q)\rangle^{1 / 2}}{\log \left(t_{j}^{4}\right)}, \quad \Lambda_{3, j}=\frac{\left\langle\partial_{q} V(q)\right\rangle^{\frac{2}{3}}}{\log \left(t_{j}^{4}\right)} \\
\Lambda_{4, j} & =\frac{t_{j}^{2}}{\log \left(t_{j}^{4}\right)}, \quad \Lambda_{5, j}=\frac{\left(1+D_{q}^{2}+R_{V}^{\geq 3}\left(q_{j}^{\prime}\right)^{4}\right)^{\frac{1}{3}}}{\log \left(t_{j}^{4}\right)} .
\end{aligned}
$$

where $t_{j} \geq 2$ we deduce

$$
\begin{aligned}
\sum_{j \notin I\left(\kappa_{3}\right)} \frac{1}{4}\left\|K_{V_{j}^{2}} u_{j}\right\|^{2}-\left(c_{d, r}^{\prime}\right)^{2} R_{V}^{\geq 3}\left(q_{j}^{\prime}\right)^{4}\left\|u_{j}\right\|^{2}+C^{(4)}\left\|u_{j}\right\|^{2} \geq \\
C^{(5)} \sum_{j \notin I\left(\kappa_{3}\right)}\left(\left\|\Lambda_{1, j} u_{j}\right\|^{2}+\left\|\Lambda_{2, j} u_{j}\right\|^{2}+\frac{1}{\kappa_{3}}\left\|\Lambda_{3, j} u_{j}\right\|^{2}+\left\|\Lambda_{4, j} u_{j}\right\|^{2}+\frac{1}{c_{0}}\left\|\Lambda_{5, j} u_{j}\right\|^{2}\right),
\end{aligned}
$$

Collecting (3.5), (3.27) and (3.28), there exists a positive constant $C^{(6)} \geq 1$ depending on $V$ such that

$$
\begin{aligned}
\left\|K_{V} u\right\|_{L^{2}}^{2}+C^{(6)}\|u\|_{L^{2}}^{2} \geq \frac{1}{C^{(6)}} \sum_{j \in \mathbb{N}}\left(\left\|\Lambda_{1, j} u_{j}\right\|^{2}+\left\|\Lambda_{2, j} u_{j}\right\|^{2}\right. & +\left\|\Lambda_{3, j} u_{j}\right\|^{2} \\
& \left.+\left\|\Lambda_{4, j} u_{j}\right\|^{2}+\left\|\Lambda_{5, j} u_{j}\right\|^{2}\right) .
\end{aligned}
$$

Step 3. In this final step, set $L(s)=\frac{s+1}{\log (s+1)}$ for all $s \geq 1$. Notice that there exists a constant $c>0$ such that for all $x \geq 1$,

$$
\inf _{t \geq 2} \frac{x}{\log (t)}+t \geq \frac{1}{c} L(x)
$$

In view of the above estimate,

$$
\begin{aligned}
\left\|\Lambda_{1, j} u_{j}\right\|^{2}+\frac{1}{4}\left\|\Lambda_{4, j} u_{j}\right\|^{2} & \geq \frac{1}{4} \int \frac{\lambda^{2}}{\left(\log \left(t_{j}^{4}\right)\right)^{2}}+t_{j}^{2} d \mu_{u_{j}}(\lambda) \\
& \geq \frac{1}{8} \int\left(\frac{\lambda}{\log \left(t_{j}\right)}+t_{j}\right)^{2} d \mu_{u_{j}}(\lambda) \\
& \geq \frac{1}{c_{3}}\left\|L\left(O_{p}\right) u_{j}\right\|^{2}
\end{aligned}
$$


Summing over $j$, we obtain the first term in the desired estimation (1.10). Likewise for the second term

$$
\left\|\Lambda_{3, j} u_{j}\right\|^{2}+\frac{1}{4}\left\|\Lambda_{4, j} u_{j}\right\|^{2} \geq \frac{1}{c_{4}}\left\|L\left(\left\langle\partial_{q} V(q)\right\rangle^{2 / 3}\right) u_{j}\right\|^{2}
$$

with

$$
\sum_{j \in \mathbb{N}}\left\|L\left(\left\langle\partial_{q} V(q)\right\rangle^{2 / 3}\right) u_{j}\right\|^{2}=\left\|L\left(\left\langle\partial_{q} V(q)\right\rangle^{2 / 3}\right) u\right\|^{2}
$$

To obtain the third term in (1.10) write samely

$$
\left\|\Lambda_{2, j} u_{j}\right\|^{2}+\frac{1}{4}\left\|\Lambda_{4, j} u_{j}\right\|^{2} \geq \frac{1}{c_{5}}\left\|L\left(\langle\operatorname{Hess} V(q)\rangle^{1 / 2}\right) u_{j}\right\|^{2}
$$

with

$$
\sum_{j \in \mathbb{N}}\left\|L\left(\langle\operatorname{Hess} V(q)\rangle^{1 / 2}\right) u_{j}\right\|^{2}=\left\|L\left(\langle\operatorname{Hess} V(q)\rangle^{1 / 2}\right) u\right\|^{2}
$$

Doing similarly again

$$
\left\|\Lambda_{5, j} u_{j}\right\|^{2}+\frac{1}{4}\left\|\Lambda_{4, j} u_{j}\right\|^{2} \geq \frac{1}{c_{6}}\left\|L\left(\left(1+D_{q}^{2}+R_{V}^{\geq 3}\left(q_{j}^{\prime}\right)^{4}\right)^{\frac{1}{3}}\right) u_{j}\right\|^{2} .
$$

By Lemma 2.5 we get

$$
\sum_{j \in \mathbb{N}}\left\|L\left(\left(1+D_{q}^{2}+R_{V}^{\geq 3}\left(q_{j}^{\prime}\right)^{4}\right)^{\frac{1}{3}}\right) u_{j}\right\|^{2} \geq \frac{1}{c_{6}}\left\|L\left(\left(1+D_{q}^{2}+R_{V}^{\geq 3}(q)^{4}\right)^{\frac{1}{3}}\right) u\right\|^{2},
$$

To conclude, just remark that

$$
\left\langle u,\left(1+D_{q}^{2}+R_{V}^{\geq 3}(q)^{4}\right) u\right\rangle \geq\left\langle u,\left(1+D_{q}^{2}\right) u\right\rangle \geq\left\langle u,\left\langle D_{q}^{2}\right\rangle u\right\rangle>\|u\|^{2}
$$

holds for all $u \in \mathcal{C}_{0}^{\infty}\left(\mathbb{R}^{2 d}\right)$, then applying (2.15) in Lemma 2.4 with $A=\left(1+D_{q}^{2}+R_{V}^{\geq 3}(q)^{4}\right)$, $B=\left\langle D_{q}^{2}\right\rangle, \alpha_{0}=\frac{2}{3}$ and $k=2$ we obtain

$$
\left\|L\left(\left(1+D_{q}^{2}+R_{V}^{\geq 3}(q)^{4}\right)^{\frac{1}{3}}\right) u\right\|^{2} \geq\left\|L\left(\left\langle D_{q}^{2}\right\rangle^{\frac{1}{3}}\right) u\right\|^{2} \geq \frac{1}{c_{7}}\left\|L\left(\left\langle D_{q}\right\rangle^{2 / 3}\right) u\right\|^{3}
$$

for all $u \in \mathcal{C}_{0}^{\infty}\left(\mathbb{R}^{2 d}\right)$.

Finally collecting all terms, we have found $C_{V} \geq 1$ such that

$$
\begin{aligned}
\left\|K_{V} u\right\|_{L^{2}}^{2}+C_{V}\|u\|_{L^{2}}^{2} \geq \frac{1}{C_{V}}\left(\left\|L\left(O_{p}\right) u\right\|_{L^{2}}^{2}\right. & +\left\|L\left(\langle\nabla V(q)\rangle^{\frac{2}{3}}\right) u\right\|_{L^{2}}^{2} \\
& \left.+\left\|L\left(\langle\operatorname{Hess} V(q)\rangle^{\frac{1}{2}}\right) u\right\|_{L^{2}}^{2}+\left\|L\left(\left\langle D_{q}\right\rangle^{\frac{2}{3}}\right) u\right\|_{L^{2}}^{2}\right)
\end{aligned}
$$

holds for all $u \in \mathcal{C}_{0}^{\infty}\left(\mathbb{R}^{2 d}\right)$. Because $\mathcal{C}_{0}^{\infty}\left(\mathbb{R}^{2 d}\right)$ is dense in $D\left(K_{V}\right)$ endowed with the graph norm, the result extends to any $u \in D\left(K_{V}\right)$. 


\section{Applications}

This section is devoted to some applications of Theorem 1.1. In each of the following examples we examine that the Assumption 1 is well fulfilled.

Example 1: Let us consider as a first example of application the case

$$
V\left(q_{1}, q_{2}\right)=-q_{1}^{2} q_{2}^{2}, \text { with } q=\left(q_{1}, q_{2}\right) \in \mathbb{R}^{2},
$$

By direct computation

$$
\partial_{q} V(q)=\left(\begin{array}{l}
-2 q_{1} q_{2}^{2} \\
-2 q_{2} q_{1}^{2}
\end{array}\right),\left|\partial_{q} V(q)\right|=2\left|q_{1} q_{2}\right||q|
$$

$$
\begin{gathered}
\text { Hess } V(q)=\left(\begin{array}{cc}
-2 q_{2}^{2} & -4 q_{1} q_{2} \\
-4 q_{1} q_{2} & -2 q_{1}^{2}
\end{array}\right),|\operatorname{Hess} V(q)|=2 \sqrt{|q|^{4}+6 q_{1}^{2} q_{2}^{2}} \asymp|q|^{2}, \\
R_{V}^{23}(q)=\left|4 q_{2}\right|^{1 / 3}+\left|4 q_{1}\right|^{1 / 3}+2 \times 4^{1 / 4} .
\end{gathered}
$$

It is clear that the trace of Hess $V(q)$ given by $-2|q|^{2}$ is stricly negative for all $q \in \mathbb{R}^{2}$. Hence

$$
\operatorname{Tr}_{-, V}(q) \geq \operatorname{Tr}_{+, V}(q) \text { for all } q \in \mathbb{R}^{2} .
$$

Moreover, for all $\kappa>0$ the algebraic set $\mathbb{R}^{2} \backslash \Sigma(\kappa)$ is not bounded since $\left(0, q_{2}\right) \in \mathbb{R}^{2} \backslash \Sigma(\kappa)$ for all $q_{2} \in \mathbb{R}$. Furthermore for $\kappa>1$ chosen as we want

$$
\lim _{\substack{q \rightarrow \infty \\ q \in \mathbb{R}^{2} \backslash \Sigma(\kappa)}} \frac{R_{V}^{23}(q)^{4}}{|\operatorname{Hess} V(q)|}=\lim _{\substack{q \rightarrow \infty \\ q \in \mathbb{R}^{2} \backslash \Sigma(\kappa)}} \frac{|q|^{4 / 3}}{|q|^{2}}=0,
$$

since $R_{V}^{\geq 3}(q)^{4} \leq|q|^{4 / 3}$ when $|q| \geq 2^{3} \times 4^{3 / 4}$.

Below we sketch as example $\Sigma(800)$ in a blue color.

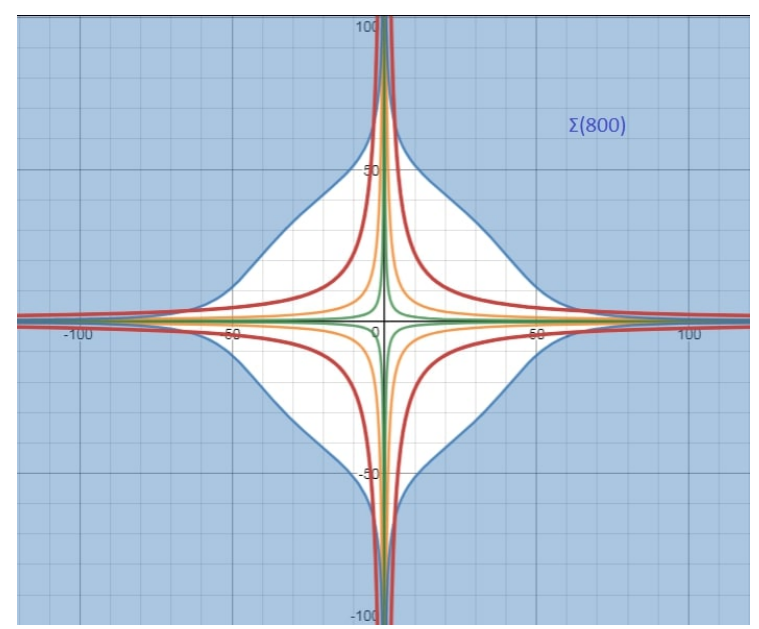

Figure 1: Contour lines of $V\left(q_{1}, q_{2}\right)=-q_{1}^{2} q_{2}^{2}$ 
Example 2: Let $n \in \mathbb{N}$. The polynomial $V(q)=-q_{1}^{2}\left(q_{1}^{2}+q_{2}^{2}\right)^{n}$ verifies the Assumption 1 only for $n=1$.

A straight forward computation shows that

$$
\partial_{q} V(q)=-\left(\begin{array}{c}
2 q_{1}\left(|q|^{2 n}+n q_{1}^{2}|q|^{2(n-1)}\right) \\
2 n q_{2} q_{1}^{2}|q|^{2(n-1)}
\end{array}\right)
$$

$$
\text { Hess } V(q)=-2|q|^{2(n-2)}\left(\begin{array}{cc}
|q|^{4}+5 n q_{1}^{2}|q|^{2}+2 n(n-1) q_{1}^{4} & 2 n q_{1} q_{2}|q|^{2}+2 n(n-1) q_{1}^{3} q_{2} \\
2 n q_{1} q_{2}|q|^{2}+2 n(n-1) q_{1}^{3} q_{2} & n q_{1}^{2}|q|^{2}+2 n(n-1) q_{1}^{2} q_{2}^{2}
\end{array}\right) \text {. }
$$

Notice that the trace of Hess $V(q)$ equals

$$
-2|q|^{2(n-2)}\left(|q|^{4}+5 n q_{1}^{2}|q|^{2}+2 n(n-1) q_{1}^{4}+n q_{1}^{2}|q|^{2}+2 n(n-1) q_{1}^{2} q_{2}^{2}\right) \leq 0
$$

for all $q \in \mathbb{R}^{2}$. Hence

$$
-\operatorname{Tr}_{-, V}(q)+\operatorname{Tr}_{+, V}(q) \leq 0, \text { for any } q \in \mathbb{R}^{2}
$$

In addition for all $\kappa>0$ the set $\mathbb{R}^{2} \backslash \Sigma(\kappa)$ is not bounded since $\left(0, q_{2}\right) \in \mathbb{R}^{2} \backslash \Sigma(\kappa)$ for all $q_{2} \in \mathbb{R}$.

For $q$ large enough $\mid$ Hess $\left.V(q)|\asymp| q\right|^{2 n}$ and $\left|D^{3} V(q)\right| \asymp|q|^{2 n-1}$ then

$$
\frac{R_{V}^{\geq 3}(q)^{4}}{|\operatorname{Hess} V(q)|} \asymp \frac{\left(|q|^{2 n-1}\right)^{4 / 3}}{|q|^{2 n}} .
$$

Hence

$$
\lim _{\substack{q \rightarrow \infty \\ q \in \mathbb{R}^{2} \backslash \Sigma(\kappa)}} \frac{R_{V}^{\geq 3}(q)^{4}}{|\operatorname{Hess} V(q)|}=0 \quad \text { if and only if } n<2 .
$$

Taking as example $\kappa=800$, we get the following shape of $\Sigma(800)$ colored in blue. 


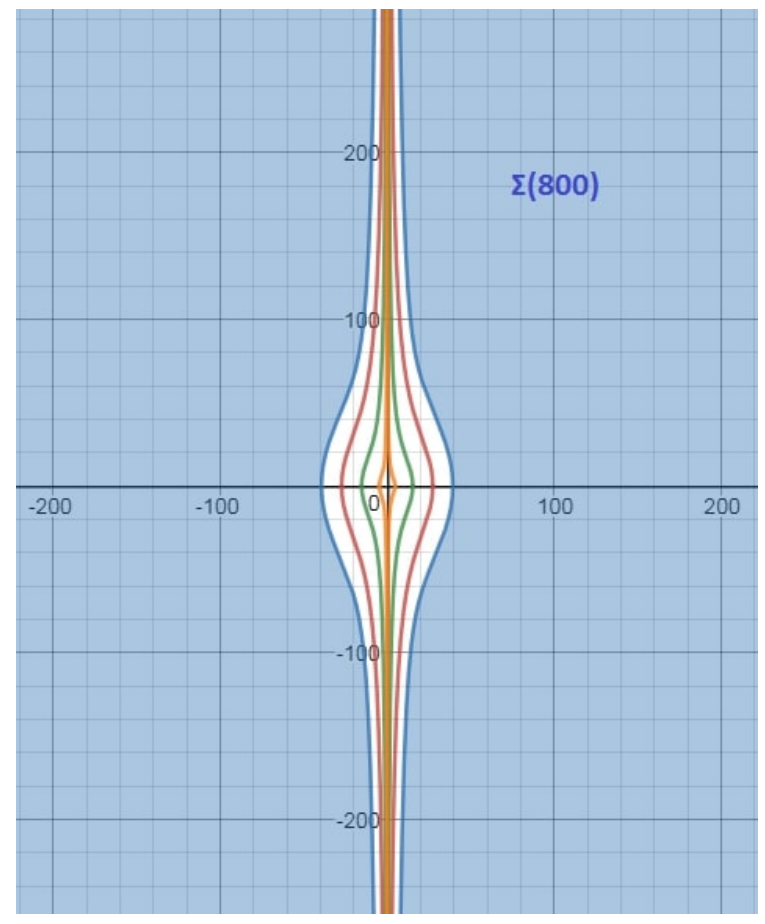

Figure 2: Contour lines of $V\left(q_{1}, q_{2}\right)=-q_{1}^{2}\left(q_{1}^{2}+q_{2}^{2}\right)$

Example 3: For $\epsilon \in \mathbb{R} \backslash\{0,-1\}$, we consider $V\left(q_{1}, q_{2}\right)=\left(q_{1}^{2}-q_{2}\right)^{2}+\epsilon q_{2}^{2}$. For all $q \in \mathbb{R}^{2}$ one has

$$
\begin{gathered}
\partial_{q} V(q)=\left(\begin{array}{c}
4 q_{1}\left(q_{1}^{2}-q_{2}\right) \\
-2\left(q_{1}^{2}-q_{2}\right)+2 \epsilon q_{2}
\end{array}\right),\left|\partial_{q} V(q)\right|=4\left|q_{1}\left(q_{1}^{2}-q_{2}\right)\right|+\left|-2\left(q_{1}^{2}-q_{2}\right)+2 \epsilon q_{2}\right| \\
\text { Hess } V(q)=\left(\begin{array}{cc}
12 q_{1}^{2}-4 q_{2} & -4 q_{1} \\
-4 q_{1} & 2(1+\epsilon)
\end{array}\right),|\operatorname{Hess} V(q)|=\left|12 q_{1}^{2}-4 q_{2}\right|+8\left|q_{1}\right|+4|1+\epsilon| \\
R_{V}^{\geq 3}(q)=\left(24\left|q_{1}\right|\right)^{1 / 3}+3 \times 4^{1 / 3}+24^{1 / 4} .
\end{gathered}
$$

In this case, we are going to show that for all $\kappa>0$ the algebraic set $\mathbb{R}^{2} \backslash \Sigma(\kappa)$ is bounded. Let $\left(q_{1}, q_{2}\right) \in \mathbb{R}^{2} \backslash \Sigma(\kappa)$ then

$$
\left(|\operatorname{Hess} V(q)|+R_{V}^{\geq 3}(q)^{4}+1\right) \geq \frac{1}{\kappa}|\nabla V(q)|^{\frac{4}{3}} .
$$

Up to a change of coordinates $X_{1}=q_{1}, X_{2}=q_{1}^{2}-q_{2}$ the above inequality is equivalent to

$$
\begin{aligned}
\left(4\left|2 X_{1}^{2}+X_{2}\right|+8\left|X_{1}\right|+4|1+\epsilon|\right. & \left.+\left(\left(24\left|X_{1}\right|\right)^{1 / 3}+3 \times 4^{1 / 3}+24^{1 / 4}\right)^{4}+1\right) \\
& \geq \frac{1}{\kappa}\left(4\left|X_{1} X_{2}\right|+\left|-2(1+\epsilon) X_{2}+2 \epsilon X_{1}^{2}\right|\right)^{\frac{4}{3}} .
\end{aligned}
$$


Using the triange inequality in the right hand side and the reverse triangle inequality with the elementary inequality $(u+v)^{\frac{4}{3}} \geq u^{\frac{4}{3}}+v^{\frac{4}{3}}$ satisfied for all $u, v \geq 0$, it follows that

$$
\left|X_{1}\right|^{2}+\left|X_{2}\right|+\left|X_{1}\right|+\left(\left|X_{1}\right|^{\frac{1}{3}}+c\right)^{4} \geq \frac{c^{\prime}}{\kappa}\left(|| 2(1+\epsilon) X_{2}|-| 2 \epsilon X_{1}^{2}||^{\frac{4}{3}}+\left|X_{1} X_{2}\right|^{\frac{4}{3}}\right) .
$$

Suppose first that $\left|X_{1}\right| \leq 1$. The inequality (4.1) implies

$$
\left|X_{2}\right|+c_{1} \geq \frac{c^{\prime}}{\kappa}|| 2(1+\epsilon) X_{2}|-| 2 \epsilon X_{1}^{2}||^{\frac{4}{3}}
$$

The right hand part in the above inequality is upper bounded by $\left|X_{2}\right|+c_{1}$ where $c_{1}$ is some positive constant. Now we distinguish two case:

Case 1: If $\frac{1}{2}\left|2(1+\epsilon) X_{2}\right| \leq\left|2 \epsilon X_{1}^{2}\right|$ or equivalently $\left|X_{2}\right| \leq\left|\frac{2 \epsilon}{1+\epsilon}\right|\left|X_{1}^{2}\right|$ then $\left|X_{2}\right| \leq\left|\frac{2 \epsilon}{1+\epsilon}\right|$.

Case 2: Else if $\frac{1}{2}\left|2(1+\epsilon) X_{2}\right| \geq\left|2 \epsilon X_{1}^{2}\right|$ then we get

$$
\left|X_{2}\right|+c_{1} \geq \frac{c^{\prime}}{\kappa}|1+\epsilon|\left|X_{2}\right|^{4 / 3} .
$$

Using the fact that $\epsilon \neq-1$, we deduce that $X_{2}$ must be also bounded.

Now if $\left|X_{1}\right| \geq 1$, we derive from (4.1) the following esimates

$$
\begin{gathered}
\left|X_{1}\right|^{2}+\left|X_{2}\right|+c_{3} \geq \frac{c_{4}}{\kappa}|| 2(1+\epsilon) X_{2}|-| 2 \epsilon X_{1}^{2}||^{\frac{4}{3}} \\
\left|X_{1}\right|^{2}+\left|X_{2}\right|+c_{3} \geq \frac{c_{4}}{\kappa}\left|X_{1} X_{2}\right|^{\frac{4}{3}} .
\end{gathered}
$$

Here we study three cases.

- Firstly if $\frac{1}{2}\left|2(1+\epsilon) X_{2}\right| \geq\left|2 \epsilon X_{1}^{2}\right|$ or equivalently $\left|X_{1}\right| \leq\left|\frac{1+\epsilon}{2 \epsilon}\right|\left|X_{2}\right|$ then (4.3) gives

$$
\left(1+\left|\frac{1+\epsilon}{\epsilon}\right|\right)\left|X_{2}\right|+c_{3} \geq \frac{c_{4}}{\kappa}\left|(1+\epsilon) X_{2}\right|^{\frac{4}{3}}
$$

Since $\epsilon \neq-1$, it follows that $X_{2}$ is bounded and so is $X_{1}$.

- Now if $2\left|2(1+\epsilon) X_{2}\right| \leq\left|2 \epsilon X_{1}^{2}\right|$ or samely $\left|X_{2}\right| \leq\left|\frac{\epsilon}{2(1+\epsilon)}\right|\left|X_{1}^{2}\right|$ the estimates (4.3) leads to

$$
\left(1+\left|\frac{\epsilon}{2(1+\epsilon)}\right|\right)\left|X_{1}\right|^{2}+c_{3} \geq \frac{c_{4}}{\kappa}\left|\epsilon X_{1}\right|^{\frac{8}{3}} .
$$

Since $\epsilon \neq 0$, it follows that $X_{1}$ is bounded and so is $X_{2}$.

- Finally if $\frac{1}{2}\left|2(1+\epsilon) X_{2}\right| \leq\left|2 \epsilon X_{1}^{2}\right| \leq 2\left|2(1+\epsilon) X_{2}\right|$, then by $(4.4)$

$$
\left(1+\left|\frac{2 \epsilon}{1+\epsilon}\right|\right)\left|X_{1}\right|^{2}+c_{3} \geq \frac{c_{4}}{\kappa}\left(\left|X_{1}\right|\left|\frac{\epsilon}{2(1+\epsilon)}\right| X_{1}^{2} \mid\right)^{\frac{4}{3}} .
$$

Hence since $\epsilon \neq 0, X_{1}$ is bounded and then $X_{2}$ is so.

Below we sketch as example $\Sigma(2)$ in a blue color. 


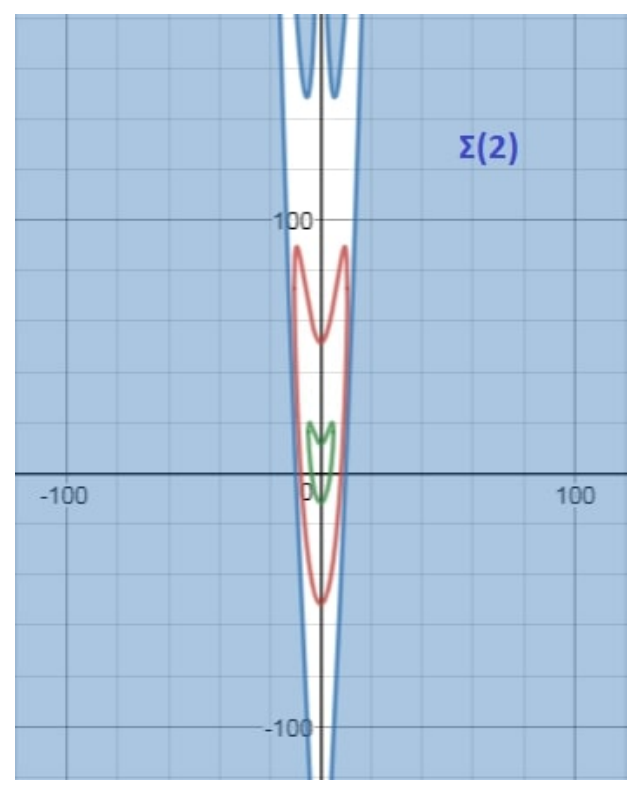

Figure 3: Contour lines of $V\left(q_{1}, q_{2}\right)=\left(q_{1}^{2}-q_{2}\right)^{2}+0.5 q_{2}^{2}$.

For $\epsilon=0$, thanks to [HeNi] (see Proposition 10.21 page 111), we know that the Witten Laplacian defined by

$$
\Delta_{V}^{(0)}=-\Delta_{q}+|\nabla V(q)|^{2}-\Delta V(q), q=\left(x_{1}, x_{2}\right) \in \mathbb{R}^{2}
$$

has no compact resolvent and then the Kramers-Fokker-Planck operator $K_{V}$ has no compact resolvent.

This example was studied in the case of the Witten Laplacian operator by B.Helffer and F.Nier in their book [HeNi]. A small mistake was done in [HeNi] in Proposition 10.20. In fact the equations $l_{11}=l_{12}=l_{111}=0$ should be replaced by $(1+\epsilon) l_{11}=l_{12}=l_{111}=0$. When $\epsilon=-1$, we can eventually construct a Weyl sequence for the Witten Laplacian operator in the following way. In this case the potential $V\left(q_{1}, q_{2}\right)=\left(q_{1}^{2}-q_{2}\right)^{2}-q_{2}^{2}$ is equal to $-2 q_{2} q_{1}^{2}+q_{1}^{4}$.

In order to construct a Weyl sequence for $\Delta_{V}^{(0)}$, it is sufficient to take $\chi\left(\frac{\left(q_{2}+n^{2}\right)}{n}\right)$ where $\chi$ is a cutoff function supported in $[-1,1]$ and then consider the sequence

$$
u_{n}\left(q_{1}, q_{2}\right)=\chi\left(\frac{\left(q_{2}+n^{2}\right)}{n}\right) \exp \left(-V\left(q_{1}, q_{2}\right)\right) .
$$

The support of $u_{n}$ is then included in $-n^{2}-n \leq q_{2} \leq-n^{2}+n$. Hence the $u_{n}$ 's have disjoint supports for large $n$.

Therefore we have

$$
-2 n^{2} \leq q_{2} \leq-\frac{n^{2}}{2} \quad \text { and } \quad-4 n^{2} q_{1}^{2}-q_{1}^{4} \leq-V\left(q_{1}, q_{2}\right) \leq-n^{2} q_{1}^{2}-q_{1}^{4} \leq-n^{2} q_{1}^{2} .
$$


As a result, we get for $n$ large

$$
\begin{aligned}
\frac{\left\langle u_{n}, \Delta_{V}^{(0)} u_{n}\right\rangle}{\left\|u_{n}\right\|^{2}} & =\frac{\left\|\left(\partial_{q}+\partial_{q} V(q)\right)\left(u_{n}\right)\right\|^{2}}{\left\|u_{n}\right\|^{2}} \\
& =\frac{\left\|\left(\partial_{q} \chi\right) e^{-V}\right\|^{2}}{\left\|u_{n}\right\|^{2}}=O\left(\frac{1}{n^{2}}\right) .
\end{aligned}
$$

Here to get the lower bound of the the above quantity we restrict the integral in $q_{1}=O\left(\frac{1}{n}\right)$. As a conclusion, for $\epsilon=-1$ the Witten Laplacian attached to $V\left(q_{1}, q_{2}\right)=q_{1}^{2} q_{2}^{2}+\epsilon\left(q_{1}^{2}+q_{2}^{2}\right)$ has no compact resolvent and then the Kramers-Fokker-Planck operator $K_{V}$ has no compact resolvent.

\section{A Slow metric, partition of unity}

The purpose of this appendix is to state with references or proofs the facts concerning metrics which are needed in the article. We first remind the following definitions.

Definitions A.1. A metric $g$ on $\mathbb{R}^{m}$ is called a slowly varying metric if there exists a constant $C \geq 1$ such that for all $x, y \in \mathbb{R}^{m}$ satisfying $g_{x}(x-y, x-y) \leq C^{-1}$ it follows that

$$
C^{-1} g_{x}(z, z) \leq g_{y}(z, z) \leq C g_{x}(z, z)
$$

holds for all $z \in \mathbb{R}^{m}$.

Let $g^{1}$ and $g^{2}$ be two metrics. We say that $g^{1}$ is $g^{2}$ slow if there is a constant $c \geq 1$ such that for all $x, y \in \mathbb{R}^{m}$

$$
g_{x}^{2}(x-y, x-y) \leq c^{-1} \Rightarrow c^{-1} g_{x}^{1}(z, z) \leq g_{y}^{1}(z, z) \leq c g_{x}^{1}(z, z) .
$$

holds for all $z \in \mathbb{R}^{m}$.

Remark A.2. The second statement in the above definitions is a typical application of the notion of the second microlocalisation developed by Bony-lerner (see [BoLe]).

Remark A.3. The property A.1 will be satisfied if we ask only that

$$
\exists C \geq 1, \forall x, y, z \in \mathbb{R}^{m}, g_{x}(x-y) \leq C^{-1} \Longrightarrow g_{y}(z) \leq C g_{x}(z) .
$$

Indeed, assuming (A.3) gives that wherever $g_{x}(x-y) \leq C^{-1}$ (which is less than or equal to one since $C \geq 1$ from (A.3) with $x=y$ ) this implies $g_{y}(y-x) \leq C^{-1}$ and then $g_{x}(z) \leq C g_{y}(z)$, so that $(A .1)$ is well satisfied.

Notations A.4. For $r \in \mathbb{N}$, let $E_{r}$ denote the set of polynomials with degree not greater than $r$ :

$$
E_{r}=\left\{P \in \mathbb{R}\left[X_{1}, \ldots, X_{d}\right], d^{\circ} P \leq r\right\} .
$$

For a polynomial $P \in E_{r}$ and $n \in\{1, \ldots, r\}$, the function $R_{P}^{\geq n}: \mathbb{R}^{d} \rightarrow \mathbb{R}$ is defined by

$$
R_{P}^{\geq n}(q)=\sum_{n \leq|\alpha| \leq r}\left|\partial_{q}^{\alpha} P(q)\right|^{\frac{1}{|\alpha|}} .
$$


In the present article we are mainly concerned with the metric $g^{n}=R_{P}^{\geq n}(q)^{2} d q^{2}$ where $n \in\{1, \ldots, r\}$ which satisfies the following properties.

Lemma A.5. Let $n$ a natural number in $\{1, \ldots, r\}$.

1) The metric $g^{n}$ is slow: There exists a uniform $C=C(n, r, d) \geq 1$ such that

$$
R_{P}^{\geq n}(q)\left|q-q^{\prime}\right| \leq C^{-1} \Longrightarrow\left(\frac{R_{P}^{\geq n}(q)}{R_{P}^{\geq n}\left(q^{\prime}\right)}\right)^{ \pm 1} \leq C
$$

2) The metric $g^{n-1}$ is $g^{n}$ slow: There is a constant $C^{\prime}=C^{\prime}(n, r, d) \geq 1$ so that

$$
R_{P}^{\geq n}(q)\left|q-q^{\prime}\right| \leq C^{\prime-1} \Longrightarrow\left(\frac{R_{P}^{\geq n-1}(q)}{R_{P}^{\geq n-1}\left(q^{\prime}\right)}\right)^{ \pm 1} \leq C^{\prime}
$$

Proof. Assume $n, r \in \mathbb{N}^{*}$ with $n \geq r$. Consider the map

$$
\begin{aligned}
f: E_{r} \rightarrow E_{r} / E_{n} ; & \\
P \mapsto \bar{P}: \mathbb{R}^{d} & \rightarrow \mathbb{R} \\
x & \mapsto \bar{P}(x) \sum_{n \leq|\alpha| \leq r} \frac{\partial_{x}^{\alpha} P(0)}{\alpha !}\left(\frac{x}{R_{P}^{\geq n}(0)}\right)^{\alpha} .
\end{aligned}
$$

Set $K_{n, r}:=f\left(E_{r}\right)=\left\{\bar{P} \in E_{r} / E_{n}, R_{\bar{P}}^{\geq n}(0)=1\right\}$. Assume $\bar{P} \in K_{n, r}$ and $\beta \in \mathbb{N}^{d}$ with $|\beta| \geq n$. Notice that there is a constant $c \geq 1$ (uniform with respect to $\bar{P}$ and $\beta$ ) such that for $|t| \leq c^{-1}$,

$$
\begin{aligned}
\left|\partial_{t}^{\beta} \bar{P}(t)-\partial_{t}^{\beta} \bar{P}(0)\right| & =\left|\sum_{|\alpha|=1} \frac{\partial_{t}^{\alpha+\beta} \bar{P}(0)}{\alpha !} t^{\alpha}\right| \\
& \leq \sum_{|\alpha|=1}\left|\frac{\partial_{t}^{\alpha+\beta} \bar{P}(0)}{\alpha !}\right||t| \leq d|t| .
\end{aligned}
$$

On the other hand, the application

$$
\mathbb{R}^{\mathbb{N}} \rightarrow \mathbb{R}^{\mathbb{N}},\left(u_{\beta}\right)_{n \leq|\beta| \leq r} \mapsto\left(\left|u_{\beta}\right|^{\frac{1}{|\beta|}}\right)_{n \leq|\beta| \leq r}
$$

is continuous. Then for all $\delta>0$ there exists $\eta=\eta(n, r)>0$ so that

$$
\max _{n \leq|\beta| \leq r}\left|u_{\beta}-v_{\beta}\right| \leq\left.\eta \Longrightarrow \sum_{n \leq|\beta| \leq r}|| u_{\beta}\right|^{\frac{1}{|\beta|}}-\left|v_{\beta}\right|^{\frac{1}{|\beta|}} \mid \leq \delta \text {. }
$$

Thus for all $\delta>0$ there is a strictly positive constant $C_{1}=C_{1}(n, r, d)=\min \left(\frac{\eta}{d}, c^{-1}\right) \leq 1$ so that

$$
\left|R_{\bar{P}}^{\geq n}(t)-R_{\bar{P}}^{\geq n}(0)\right| \leq \delta=\delta R_{\bar{P}}^{\geq n}(0),
$$


holds when $|t| \leq C_{1}$.

Now given a polynomial $V \in E_{r}$ and $q \in \mathbb{R}^{d}$ define

$$
\bar{P}_{q}(t)=V\left(q+R_{V}^{\geq n}(q)^{-1} t\right) .
$$

Writting

$$
R_{\bar{P}_{q}}^{\geq n}(t)=\sum_{n \leq|\alpha| \leq r}\left|\partial_{t}^{\alpha} \bar{P}_{q}(t)\right|^{\frac{1}{\alpha \mid}}=R_{V}^{\geq n}(q)^{-1} R_{V}^{\geq n}\left(q+R_{V}^{\geq n}(q)^{-1} t\right),
$$

clearly the polynomial $\bar{P}_{q}$ belongs to the set $K$. Hence for $\delta=\frac{1}{2}$ we get by (A.9)

$$
\frac{1}{2} R_{\bar{P}_{q}}^{\geq n}(0) \leq R_{\bar{P}_{q}}^{\geq n}(t) \leq 2 R_{\bar{P}_{q}}^{\geq n}(0)
$$

when $|t| \leq C_{1}$.

It follows from (A.11) and (A.11),

$$
\left(\frac{R_{V}^{\geq n}\left(q+R_{V}^{\geq n}(q)^{-1} t\right)}{R_{V}^{\geq n}(q)}\right)^{ \pm 1} \leq 2
$$

for $|t| \leq C_{1}$.

Therefore by the above inequality there is a constant $C_{1} \leq 1$ (chosen uniformly with respect to $q, V$ once $r, n$ and $d$ are fixed) so that for all $q, q^{\prime} \in \mathbb{R}^{d}$ such that $R_{V}^{\geq n}(q)\left|q^{\prime}-q\right| \leq C_{1}$,

$$
\left(\frac{R_{V}^{\geq n}\left(q^{\prime}\right)}{R_{V}^{\geq n}(q)}\right)^{ \pm 1} \leq 2
$$

It remains now to prove that for every $n \in\{1, \ldots, r\}$, the metric $g^{n-1}$ is $g^{n}$ slow. Assuming the slowlness of $g^{n}$, the inequality

$$
\left(\frac{R_{V}^{\geq n}\left(q^{\prime}\right)}{R_{V}^{\geq n}(q)}\right)^{ \pm 1} \leq 2
$$

holds when $R_{V}^{\geq n}(q)\left|q^{\prime}-q\right| \leq C_{1}^{-1}$.

Denote as before $t=R_{V}^{\geq n}(q)\left(q^{\prime}-q\right)$ and $\bar{P}_{q}(t)=V\left(q+R_{V}^{\geq n}(q)^{-1} t\right)$. Taking into account (A.14) and (A.10) it results

$$
|t| \leq C_{1}^{-1} \Longrightarrow\left|\partial_{t}^{\alpha} \bar{P}_{q}(t)\right|^{\frac{1}{|\alpha|}} \leq R_{\bar{P}_{q}}^{\geq n}(t) \leq 2,
$$

for all $\alpha \in \mathbb{N}^{d}$ with $|\alpha| \geq n$. Consequently,

$$
|t| \leq C_{1}^{-1} \Longrightarrow\left|\partial_{t}^{\alpha} \bar{P}_{q}(t)\right| \leq 2^{r},
$$

for all $\alpha \in \mathbb{N}^{d},|\alpha| \geq n$. 
Using (A.15), one has when $|\alpha|=n-1$ and $|t| \leq C_{1}^{-1}$

$$
\begin{aligned}
\left|\partial_{t}^{\alpha} \bar{P}_{q}(t)-\partial_{t}^{\alpha} \bar{P}_{q}(0)\right| & =\left|\sum_{|\beta|=1} \frac{\partial_{t}^{\beta}\left[\partial_{t}^{\alpha} \bar{P}_{q}\right](0)}{\beta !} t^{\beta}\right|=\left|\sum_{|\beta|=1} \frac{\partial_{t}^{\alpha+\beta} \bar{P}_{q}(0)}{\beta !} t^{\beta}\right| \\
& \leq \sum_{|\beta|=1}\left|\frac{\partial_{t}^{\alpha+\beta} \bar{P}_{q}(0)}{\beta !}\right||t| \leq d 2^{r}|t|
\end{aligned}
$$

since $|\alpha+\beta| \geq n$. On the other hand, the application

$$
\mathbb{R}^{\mathbb{N}} \rightarrow \mathbb{R}^{\mathbb{N}},\left(u_{\beta}\right)_{|\beta|=n-1} \mapsto\left(\left|u_{\beta}\right|^{\frac{1}{|\beta|}}\right)_{|\beta|=n-1}
$$

is continuous. Then for all $\delta>0$ there exists $\eta^{\prime}=\eta^{\prime}(n)>0$ so that

$$
\max _{|\beta|=n-1}\left|u_{\beta}-v_{\beta}\right| \leq\left.\eta^{\prime} \Longrightarrow \sum_{|\beta|=n-1}|| u_{\beta}\right|^{\frac{1}{|\beta|}}-\left|v_{\beta}\right|^{\frac{1}{\beta \mid}} \mid \leq \delta
$$

Hence for $\delta=1$ there is a strictly positive constant $C_{1}^{\prime}=C_{1}^{\prime}(n, r, d)=\min \left(\frac{\eta^{\prime}}{d 2^{r}}, C_{1}^{-1}\right) \leq 1$ so that

$$
\left.\left|\sum_{|\alpha|=n-1}\right| \partial_{t}^{\alpha} \bar{P}_{q}(t)\right|^{\frac{1}{|\alpha|}}-\left|\partial_{t}^{\alpha} \bar{P}_{q}(0)\right|^{\frac{1}{|\alpha|}} \mid \leq 1
$$

holds when $|t| \leq C_{1}^{\prime}$.

Using respectively Peetre's inequality $\left(\left(\frac{\left\langle X^{\prime}\right\rangle}{\langle X\rangle}\right)^{s} \leq 2^{\frac{|s|}{2}}\left\langle X-X^{\prime}\right\rangle\right.$ for all $\left.s \in \mathbb{R}, X, X^{\prime} \in \mathbb{R}\right)$ then (A.18) yields when $|t| \leq C_{1}^{\prime}$

$$
\left(\frac{\left\langle\sum_{|\alpha|=n-1}\left|\partial_{t}^{\alpha} \bar{P}_{q}(t)\right|^{\frac{1}{|\alpha|}}\right\rangle}{\left\langle\sum_{|\alpha|=n-1}\left|\partial_{t}^{\alpha} \bar{P}_{q}(0)\right|^{\frac{1}{|\alpha|}}\right\rangle}\right)^{ \pm 1} \leq \sqrt{2}\left\langle\sum_{|\alpha|=n-1}\left|\partial_{t}^{\alpha} \bar{P}_{q}(t)\right|^{\frac{1}{|\alpha|}}-\left|\partial_{t}^{\alpha} \bar{P}_{q}(0)\right|^{\frac{1}{|\alpha|}}\right\rangle \leq 2 .
$$

Remember that for any sequence $\left(a_{i}\right)_{1 \leq i \leq N}$ of positive numbers

$$
\left(\sum_{1}^{N} a_{i}^{p}\right)^{\frac{1}{p}} \leq \sum_{1}^{N} a_{i} \leq N^{\frac{1}{q}}\left(\sum_{1}^{N} a_{i}^{p}\right)^{\frac{1}{p}}
$$

where the two real numbers $p, q>1$ are conjugate indices. In particular for any real numbers $a, b$

$$
\left(a^{2}+b^{2}\right)^{\frac{1}{2}} \leq(|a|+|b|) \leq 2^{2}\left(a^{2}+b^{2}\right)^{\frac{1}{2}}
$$

It results from the elementary above inequality

$$
\left\langle\sum_{|\alpha|=n-1}\left|\partial_{t}^{\alpha} \bar{P}_{q}(t)\right|^{\frac{1}{|\alpha|}}\right\rangle \leq\left(1+\sum_{|\alpha|=n-1}\left|\partial_{t}^{\alpha} \bar{P}_{q}(t)\right|^{\frac{1}{|\alpha|}}\right) \leq 4\left\langle\sum_{|\alpha|=n-1}\left|\partial_{t}^{\alpha} \bar{P}_{q}(t)\right|^{\frac{1}{|\alpha|}}\right\rangle,
$$


and

$$
\left\langle\sum_{|\alpha|=n-1}\left|\partial_{t}^{\alpha} \bar{P}_{q}(0)\right|^{\frac{1}{|\alpha|}}\right\rangle \leq\left(1+\sum_{|\alpha|=n-1}\left|\partial_{t}^{\alpha} \bar{P}_{q}(0)\right|^{\frac{1}{|\alpha|}}\right) \leq 4\left\langle\sum_{|\alpha|=n-1}\left|\partial_{t}^{\alpha} \bar{P}_{q}(0)\right|^{\frac{1}{|\alpha|}}\right\rangle .
$$

Using the above two estimates with (A.19) we immediately get for $|t| \leq C_{1}^{\prime}$

$$
\left(\frac{1+\sum_{|\alpha|=n-1}\left|\partial_{t}^{\alpha} \bar{P}_{q}(t)\right|^{\frac{1}{|\alpha|}}}{1+\sum_{|\alpha|=n-1}\left|\partial_{t}^{\alpha} \bar{P}_{q}(0)\right|^{\frac{1}{|\alpha|}}}\right)^{ \pm 1} \leq 8
$$

Notice that by (A.14)

$$
\left(\frac{1+\sum_{|\alpha|=n-1}\left|\partial_{t}^{\alpha} \bar{P}_{q}(t)\right|^{\frac{1}{|\alpha|}}}{1+\sum_{|\alpha|=n-1}\left|\partial_{t}^{\alpha} \bar{P}_{q}(0)\right|^{\frac{1}{|\alpha|}}}\right)^{ \pm 1}=\left(\frac{1+\sum_{|\alpha|=n-1}\left(\frac{\left|\partial_{q}^{\alpha} V\left(q^{\prime}\right)\right|}{R_{V}^{\geq n}(q)^{|\alpha|}}\right)^{\frac{1}{|\alpha|}}}{1+\sum_{|\alpha|=n-1}\left(\frac{\left|\partial_{q}^{\alpha} V(q)\right|}{R_{V}^{\geq n}(q)^{|\alpha|}}\right)^{\frac{1}{|\alpha|}}}\right)^{ \pm 1} \geq \frac{1}{2}\left(\frac{R_{V}^{\geq n-1}\left(q^{\prime}\right)}{R_{V}^{\geq n-1}(q)}\right)^{ \pm 1}
$$

In conclusion, from $(\mathrm{A} .21)$ and $(\mathrm{A} .22)$ there is a constant $C_{1}^{\prime}=\min \left(\frac{\eta^{\prime}}{d 2^{r}}, C_{1}^{-1}\right) \leq 1$ so that

$$
R_{V}^{\geq n}(q)\left|q-q^{\prime}\right| \leq C_{1}^{\prime} \Longrightarrow\left(\frac{R_{V}^{\geq n-1}\left(q^{\prime}\right)}{R_{V}^{\geq n-1}(q)}\right)^{ \pm 1} \leq 16
$$

The main feature of a slow varying metric is that it is possible to introduce some partitions of unity related to the metric in a way made precise in the following theorem. For more details and proof see [Hor1] ( Section 1.4 page 25).

Theorem A.6. [Hor1] For any slowly varying metric $g$ in $\mathbb{R}^{m}$ one can choose a sequence $x_{\nu} \in \mathbb{R}^{m}$ such that the balls

$$
B_{\nu}=\left\{x ; g_{x_{\nu}}\left(x-x_{\nu}\right)<1\right\}
$$

form a covering of $\mathbb{R}^{m}$ for which the intersection of more than $N=\left(4 C^{3}+1\right)^{m}$ balls $B_{\nu}$ is always empty $(C$ is the constant in $(A .1))$. In addition, for any decreasing sequence $d_{i}$ with $\sum_{j} d_{j}=1$ one can choose non negative $\phi_{\nu} \in \mathcal{C}_{0}^{\infty}\left(B_{\nu}\right)$ with $\sum \phi_{\nu}=1$ in $\mathbb{R}^{m}$ so that for all $k$

$$
\left|\phi_{\nu}^{(k)}\left(x ; y_{1}, \cdots, y_{k}\right)\right| \leq\left(N C C_{1}\right)^{k} g_{x}\left(y_{1}, 0\right) \cdots g_{x}\left(y_{k}, 0\right) / d_{1} \cdots d_{k}
$$

where $C$ is the constant in (A.1) and $C_{1}$ is a constant that depends only on $m$.

Regarding the above Theorem we have the following result. 
Lemma A.7. Let $P \in E_{r}$ and $n \in\{1, \ldots, r\}$, then there exists a partition of unity $\sum_{j \in \mathbb{N}} \psi_{j}(q)^{2} \equiv 1$ in $\mathbb{R}^{d}$ such that:

1) For all $q \in \mathbb{R}^{d}$, the cardinality of the set $\left\{j, \Psi_{j}(q) \neq 0\right\}$ is uniformely bounded.

2) For any natural number $j \in \mathbb{N}$,

$$
\operatorname{supp} \Psi_{j} \subset B\left(q_{j}, a R_{P}^{\geq n}\left(q_{j}\right)^{-1}\right) \quad \text { and } \quad \Psi_{j} \equiv 1 \text { in } B\left(q_{j}, b R_{P}^{\geq n}\left(q_{j}\right)^{-1}\right),
$$

for some $q_{j} \in \mathbb{R}^{d}$ with $0<b<a$ independent of $j \in \mathbb{N}$.

3) For all $\alpha \in \mathbb{N}^{d} \backslash\{0\}$, there exists $c_{\alpha}>0$ such that

$$
\sum_{j \in \mathbb{N}}\left|\partial_{q}^{\alpha} \Psi_{j}\right|^{2} \leq c_{\alpha} R_{P}^{\geq n}(q)^{2|\alpha|}
$$

Moreover the constants $a, b$ et $c_{\alpha}$ can be chosen uniformly with respect to $P \in E_{r}$, once the degree $r \in \mathbb{N}$ and the dimension $d \in \mathbb{N}$ are fixed.

\section{B Around Tarski-Seidenberg theorem}

In this appendix we give an application of the Tarski-Seidemberg theorem [Hor2], which we state in the following geometric form. We first introduce a few basic concepts which are needed for the state.

Definition B.1. A subset of $\mathbb{R}^{n}$ is called semi-algebraic if it is a finite union of finite intersections of sets defined by polynomial equations or inequalities.

Definition B.2. Let $A \subset \mathbb{R}^{n}$ and $B \subset \mathbb{R}^{m}$ be two sub-algebraic sets. The function $f: A \rightarrow B$ is said to be semi-algebraic if its graph $\Gamma_{f}=\{(x, y) \in A \times B ; y=f(x)\}$ is a semi-algebraic set of $\mathbb{R}^{n} \times \mathbb{R}^{m}$.

Theorem B.3. [Hor2](Tarski-Seidenberg) If $A$ is a semi-algebraic subset of $\mathbb{R}^{n+m}=\mathbb{R}^{n} \oplus \mathbb{R}^{m}$, then the projection $A^{\prime}$ of $A$ in $\mathbb{R}^{m}$ is also semi-algebraic.

Proposition B.4. [Hor2] If $E$ is a semi-algebraic set on $\mathbb{R}^{2+n}$, and

$$
f(x)=\inf \left\{y \in \mathbb{R} ; \exists z \in \mathbb{R}^{n},(x, y, z) \in E\right\}
$$

is defined and finite for large positive $x$, then $f$ is identically 0 for lage $x$ or else

$$
f(x)=A x^{a}(1+o(1)), \quad x \rightarrow+\infty
$$

where $A \neq 0$ and a is a rational number.

We refer to [Hor2] (see Theorem A.2.2 and Theorem A.2.5) for detailed proofs of Theorem B.3 and Proposition B.4.

In the final part of this section we list and recall the following notations. 
Notation B.5. Let $P$ be a polynomial of degree $r$. For all natural number $n \in\{0, \cdots, r\}$ and every $q \in \mathbb{R}^{d}$

$$
\begin{aligned}
& R_{P}^{\geq n}(q)=\sum_{n \leq|\alpha| \leq r}\left|\partial_{q}^{\alpha} P(q)\right|^{\frac{1}{|\alpha|}}, \\
& R_{P}^{=n}(q)=\sum_{|\alpha|=n}\left|\partial_{q}^{\alpha} P(q)\right|^{\frac{1}{|\alpha|}} .
\end{aligned}
$$

Lemma B.6. Let $\Sigma$ be an unbounded semialgebraic set and $V$ a polynomial of degree $r$ satisfying the following assumption

$$
\lim _{\substack{q \rightarrow \infty \\ q \in \Sigma}} \frac{R_{V}^{\geq n}(q)^{\alpha}}{R_{V}^{=m}(q)^{2}}=0
$$

where $\alpha \in \mathbb{Q}, n, m \in\{0,1, \cdots, r-1\}$ are fixed numbers.

Then there exist $\delta \in(0,1)$ and a positive function $\Lambda_{\Sigma}:(0,+\infty) \rightarrow[0,+\infty)$ so that

$$
\begin{aligned}
& \quad \forall q \in \Sigma,|q| \geq \varrho, \quad \Lambda_{\Sigma}(\varrho) R_{V}^{\geq n}(q)^{\alpha} \leq R_{V}^{=m}(q)^{2(1-\delta)} \\
& \text { and } \quad \lim _{\varrho \rightarrow+\infty} \Lambda_{\Sigma}(\varrho)=+\infty .
\end{aligned}
$$

Proof. Suppose that there are $\alpha \in \mathbb{Q}, n, m \in\{0,1, \cdots, r-1\}$ such that

$$
\lim _{\substack{q \rightarrow \infty \\ q \in \Sigma}} \frac{R_{V}^{\geq n}(q)^{\alpha}}{R_{V}^{=m}(q)^{2}}=0
$$

where $\Sigma$ is a given unbounded semialgebraic set.

After setting $\tau=\operatorname{ppcm}(|\beta|, \min (n, m) \leq|\beta| \leq r)$, define the functions $\widetilde{R}_{V}^{\geq n}$ and $\widetilde{R}_{V}^{=m}$, for all $q \in \mathbb{R}^{d}$ by

$$
\widetilde{R}_{V}^{\geq n}(q)=\sum_{n \leq|\alpha| \leq r}\left|\partial_{q}^{\alpha} V(q)\right|^{\frac{\tau}{\alpha \mid}}
$$

and

$$
\widetilde{R}_{V}^{=m}(q)=\sum_{|\alpha|=m}\left|\partial_{q}^{\alpha} V(q)\right|^{\frac{\tau}{\alpha \mid}}
$$

Notice that one has the equivalences $R_{V}^{\geq n}(q) \asymp\left(\widetilde{R}_{V}^{\geq n}(q)\right)^{\frac{1}{\tau}}$ and $R_{V}^{=m}(q) \asymp\left(\widetilde{R}_{V}^{=m}(q)\right)^{\frac{1}{\tau}}$ for all $q \in \mathbb{R}^{d}$ where the functions $R_{V}^{\geq n}$ and $R_{V}^{=m}$ are defined respectively as in (B.1) and (B.2). Clearly the Assumption (B.4) is equivalent to

$$
\lim _{\substack{q \rightarrow \infty \\ q \in \Sigma}} \frac{\widetilde{R}_{V}^{\geq n}(q)^{\alpha}}{\widetilde{R}_{V}^{=m}(q)^{2}}=0 .
$$


Remark here that $\widetilde{R}_{V}^{\geq n}(q)$ and $\widetilde{R}_{V}^{=m}(q)$ are polynomials in $q \in \mathbb{R}^{d}$ variable. Furthermore, the Assumption (B.5) can be written as follows

$$
\widetilde{R}_{V}^{\geq n}(q)^{\alpha} \leq \epsilon(q) \widetilde{R}_{V}^{=m}(q)^{2},
$$

for all $q \in \Sigma$ where

$$
\epsilon(q)=\inf \left\{\epsilon>0, \epsilon \widetilde{R}_{V}^{=m}(q)^{2}-\widetilde{R}_{V}^{\geq n}(q)^{\alpha}>0\right\}, \quad \lim _{\substack{q \rightarrow \infty \\ q \in \Sigma}} \epsilon(q)=0 .
$$

Now, following the notations of Proposition B.4, we introduce the set

$$
E=\left\{(q, \varrho, \epsilon) \in \mathbb{R}^{d+2} \text { such that } \epsilon \widetilde{R}_{V}^{=m}(q)^{2}-\widetilde{R}_{V}^{\geq n}(q)^{\alpha}>0 \text { and }|q|^{2} \geq \varrho^{2}\right\},
$$

and the function $f$ defined in $\mathbb{R}_{+}$by

$$
f(\varrho)=\inf \{\epsilon>0, \operatorname{such} \text { that }(q, \varrho, \epsilon) \in E\} .
$$

By Tarski-Seidenberg theorem (see Theorem B.3), the function $f$ is semialgebraic in $\varrho$. Moreover $f$ is defined, finite and not identically zero. Then by Proposition B.4, there exist a constant $A>0$ and a rational number $\gamma$ such that

$$
f(\varrho)=A \varrho^{\gamma}+o_{\varrho \rightarrow+\infty}\left(\varrho^{\gamma}\right) .
$$

By the definition (B.7) and (B.6), $\lim _{\varrho \rightarrow+\infty} f(\varrho)=0$ and then $\gamma<0$. Hence for $\varrho \geq 1$, we know $f(\varrho) \leq \frac{2 A}{\varrho^{|\gamma|}}$. We deduce for $|q| \geq 1$,

$$
\widetilde{R}_{V}^{\geq n}(q)^{\alpha} \leq f(|q|) \widetilde{R}_{V}^{=m}(q)^{2} \leq \frac{2 A}{|q||\gamma|} \widetilde{R}_{V}^{=m}(q)^{2}
$$

and

$$
\frac{|q|^{|\gamma| / 2}}{2 A} \widetilde{R}_{V}^{\geq n}(q)^{\alpha} \leq \frac{1}{|q|^{\frac{|\gamma|}{2}}} \widetilde{R}_{V}^{=m}(q)^{2} .
$$

In particular, since $\widetilde{R}_{V}^{\geq n}(q) \geq \widetilde{R}_{V}^{=r}(0)>0, \widetilde{R}_{V}^{=m}(q)$ does not vanish for $q \in \Sigma$ with $|q| \geq 1$.

On the other hand, notice

$$
\forall q \in \Sigma,|q| \geq 1, \quad \widetilde{R}_{V}^{=m}(q) \leq c|q|^{\tau r} .
$$

The inequalities (B.8) and (B.10) lead to

$$
\widetilde{R}_{V}^{\geq n}(q)^{\alpha} \leq C|q|^{2 \tau r-|\gamma|}
$$

for every $q \in \Sigma$ with $|q| \geq \rho \geq 1$. Therefore since $\widetilde{R}_{V}^{\geq n}(q) \geq \widetilde{R}_{V}^{=r}(0)>0$ we deduce $|\gamma| \leq 2 \tau r$. Using again (B.10) we get

$$
\frac{1}{|q|^{\frac{|\gamma|}{2}}} \leq \frac{c^{\frac{|\gamma|}{2 \tau r}}}{\widetilde{R}_{V}^{=m}(q)^{\frac{|\gamma|}{2 \tau r}}}
$$


for any $q \in \Sigma$ with $|q| \geq 1$.

From (B.9) and (B.11), we deduce

$$
\forall q \in \Sigma,|q| \geq \varrho \geq 1, \quad \frac{\varrho^{|\gamma| / 2}}{2 A} \widetilde{R}_{V}^{\geq n}(q)^{\alpha} \leq \frac{|q|^{|\gamma| / 2}}{2 A} \widetilde{R}_{V}^{\geq n}(q)^{\alpha} \leq c^{\frac{|\gamma| \gamma \mid}{2 r}} \widetilde{R}_{V}^{=m}(q)^{2\left(1-\frac{|\gamma|}{4 \tau r}\right)} .
$$

We now take $\delta=\frac{|\gamma|}{4 \tau r} \in(0,1)$ and

$$
\Lambda_{\Sigma}(\varrho)= \begin{cases}\frac{\varrho^{|\gamma| / 2}}{2 A \frac{|\gamma|}{2 \tau r}} & \text { if } \varrho \geq 1 \\ 0 & \text { else }\end{cases}
$$

Acknowledgement I express my sincere gratitude to Professor Francis Nier. As a PhD advisor, Professor Nier supported me in this work.

\section{References}

[AlVi] A. Aleman, J. Viola: On weak and strong solution operators for evolution equations coming from quadratic operators. J. Spectr. Theory 8, no. 1, 33-121, (2018).

[BNV] M. Ben Said, F. Nier, J. Viola : Quaternionic structure and analysis of some KramersFokker-Planck. Arxiv1807.01881, (2018).

[BoLe] J.-M. Bony; N. Lerner: Quantification asymptotique et microlocalisations d'ordre suprieur. Ann. Sc. ENS, 22, 377-433, (1989).

[HeNi] B. Helffer, F. Nier: Hypoelliptic estimates and spectral theory for Fokker-Planck operators and Witten Laplacians. Lecture Notes in Mathematics, 1862. Springer-Verlag. $\mathrm{x}+209 \mathrm{pp},(2005)$

[HeNo] B. Helffer, J. Nourrigat: Hypoellipticité maximale pour des opérateurs polynômes de champs de vecteurs. Progress in Mathematics, 58, (1985).

[HerNi] F. Hérau, F. Nier: Isotropic hypoellipticity and trend to equilibrium for the FokkerPlanck equation with a high-degree potential. Arch. Ration. Mech. Anal. 171, no. 2, 151-218, (2004).

[HiPr] M. Hitrik, K. Pravda-Starov: Spectra and semigroup smoothing for non-elliptic quadratic operators. Math. Ann. 344, no. 4, 801846, (2009).

[Hor] L. Hörmander: Symplectic classification of quadratic forms, and general Mehler formulas. Math. Z., 219:413-449, (1995).

[Hor1] L. Hörmander: The analysis of linear partial differential operators. I. Distribution theory and Fourier analysis. Reprint of the second (1990) edition [Springer, Berlin; MR1065993]. Classics in Mathematics. Springer-Verlag, Berlin. x+440 pp, (2003). 
[Hor2] L. Hörmander: The analysis of linear partial differential operators. II. Differential operators with constant coefficients. Reprint of the 1983 original. Classics in Mathematics. Springer-Verlag, Berlin. viii+392 pp, (2005).

[Li] W.-X. Li: Global hypoellipticity and compactness of resolvent for Fokker-Planck operator. Ann. Sc. Norm. Super. Pisa Cl. Sci. (5) 11 , no. 4, 789-815, (2012).

[Li2] W.-X. Li: Compactness criteria for the resolvent of Fokker-Planck operator.prepublication. ArXiv1510.01567, (2015).

[Nie] F. Nier: Hypoellipticity for Fokker-Planck operators and Witten Laplacians, "Lectures on the analysis of nonlinear partial differential equations", Morningside Lect. Math., 1, Int. Press, Somerville, MA, Part 1, 3184, (2012).

[Nou] J. Nourrigat: Subelliptic estimates for systems of pseudo-differential operators. Course in Recife. University of Recife, (1982).

[Sim] B. Simon: Convexity: An Analytic Viewpoint, Cambridge Tracts in Mathematics 187, Cambridge University Press, Cambridge, (2011)

[Vil] Cédric Villani: Hypocoercivity. Memoirs of the American Mathematical Society. 202 no. 950, iv+141 pp, (2009).

[Vio] J. Viola: Spectral projections and resolvent bounds for partially elliptic quadratic differential operators. J. Pseudo-Diff. Oper. Appl. 4, no. 2, 145-221, (2013).

[Vio1] J. Viola: The elliptic evolution of non-self-adjoint degree-2 Hamiltonians. ArXiv 1701.00801, (2017). 